Supporting information for:

Single and Double Hydroboration of B-B Triple Bonds and Convergent Routes to a Cationic Tetraborane

Tobias Brückner, ${ }^{1,2}$ Tom E. Stennett, ${ }^{1,2}$ Merlin Heß ${ }^{1,2}$ and Holger Braunschweig ${ }^{1,2}$

${ }^{1}$ Institute for Inorganic Chemistry, Julius-Maximilians-Universität Würzburg, Am Hubland, 97074 Würzburg (Germany)

${ }^{2}$ Institute for Sustainable Chemistry \& Catalysis with Boron, Julius-Maximilians-Universität Würzburg, Am Hubland, 97074 Würzburg (Germany)

\title{
Contents
}

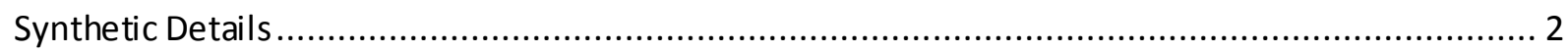

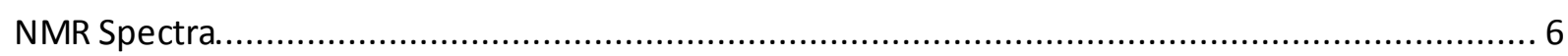

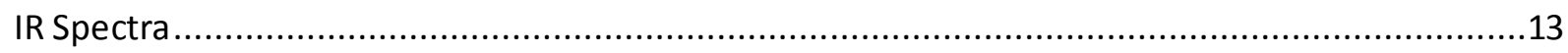

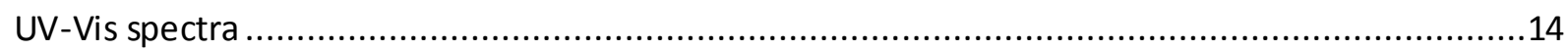

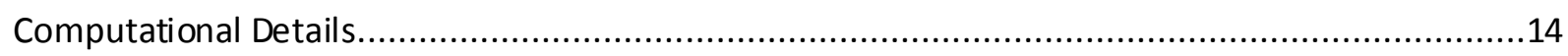

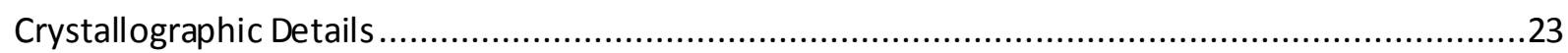

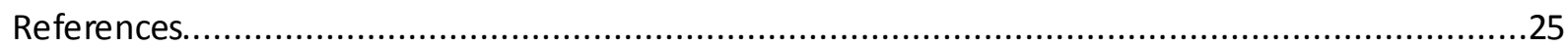




\section{Synthetic Details}

General Considerations. All manipulations were performed either under an atmosphere of dry argon or in vacuo using standard Schlenk line or glovebox techniques. Deuterated solvents were dried over molecular sieves and degassed by three freeze-pump-thaw cycles prior to use. All other solvents were distilled and degassed from appropriate drying agents. Solvents (both deuterated and non-deuterated) were stored under argon over activated $4 \AA$ molecular sieves. NMR spectra were acquired on a Bruker Avance 500 NMR spectrometer $\left({ }^{1} \mathrm{H}: 500.1 \mathrm{MHz},{ }^{11} \mathrm{~B}: 160.5 \mathrm{MHz},{ }^{13} \mathrm{C}\left\{{ }^{1} \mathrm{H}\right\}: 125.8 \mathrm{MHz},{ }^{19} \mathrm{~F}\left\{{ }^{1} \mathrm{H}\right\}: 470.6 \mathrm{MHz}\right)$ or on a Bruker Avance 400 NMR spectrometer $\left({ }^{1} \mathrm{H}: 400.1 \mathrm{MHz},{ }^{11} \mathrm{~B}: 128.4 \mathrm{MHz},{ }^{13} \mathrm{C}\left\{{ }^{1} \mathrm{H}\right\}: 125.8 \mathrm{MHz}\right.$, $\left.{ }^{19} \mathrm{~F}\left\{{ }^{1} \mathrm{H}\right\}: 376.5 \mathrm{MHz}\right)$ at $298 \mathrm{~K}$ unless otherwise stated. Chemical shifts $(\delta)$ are given in ppm and internally referenced to the carbon nuclei $\left({ }^{13} \mathrm{C}\left\{{ }^{1} \mathrm{H}\right\}\right)$ or residual protons $\left({ }^{1} \mathrm{H}\right)$ of the solvent. ${ }^{11} \mathrm{~B} \mathrm{NMR}$ spectra were referenced to $\left[\mathrm{BF}_{3} \cdot \mathrm{OEt}_{2}\right]$ as an external standard. High-resolution mass spectrometry data was obtained from a Thermo Scientific Exactive Plus spectrometer in ASAP or LIFDI mode. Elemental analysis was conducted on an Elementar Vario MICRO cube elemental analyser. UV/Vis spectra were acquired on a JASCO-V660 UV/Vis spectrometer under inert conditions inside a glovebox. IR spectra were recorded with a Bruker Alpha spectrometer with an apodized resolution of $1 \mathrm{~cm}^{-1}$ in the attenuated total reflection (ATR) mode in the region of $4000-400 \mathrm{~cm}^{-1}$ using a setup with a diamond crystal. $\mathrm{HBcat}$ and MeOTf were purchased from Sigma Aldrich. SIDipMes, ${ }^{1} \mathrm{~B}_{2}(\text { SIDipMes })_{2}{ }^{2}$ and $\mathrm{B}_{2}(\text { SIDipMes })_{2}(\text { Bcat })_{2}(\mathbf{5})^{3}$ were synthesized following literature procedures. 


\section{Synthesis of (SIDipMes)(H)B=B(Bcat)(SIDipMes) (2)}

$\mathrm{B}_{2}(\text { SIDipMes })_{2}\left(1^{\text {SIDipMes }}, 30.0 \mathrm{mg}, 41.9 \mu \mathrm{mol}\right)$ was dissolved in benzene $(0.5 \mathrm{~mL})$ and treated with $\mathrm{HBcat}(4.5 \mathrm{mg}, 37.2 \mu \mathrm{mol})$, and within $1 \mathrm{~min}$ the solution turned from red to violet. All volatiles were evaporated in vacuo and the product was extracted with hexane. The recrystallized product was washed twice with $0.5 \mathrm{~mL}$ cold hexane and dried in vacuo. The product 2 was isolated in $32 \%$ yield as a red solid. Crystals suitable for $\mathrm{X}$-ray diffraction were obtained by slow evaporation of a saturated pentane solution.

${ }^{1} \mathrm{H}\left\{{ }^{11} \mathrm{~B}\right\} \operatorname{NMR}\left(500.1 \mathrm{MHz}, \mathrm{C}_{6} \mathrm{D}_{6}, 298 \mathrm{~K}\right): 7.22-7.19\left(\mathrm{~m}, 1 \mathrm{H}, \mathrm{CH}_{\mathrm{Ar}}\right), 7.11-7.08\left(\mathrm{~m}, 3 \mathrm{H}, \mathrm{CH}_{\mathrm{Ar}}\right)$, 6.98-6.96 (m, 2H, CH $\left.H_{\text {Ar }}\right), 6.90-6.85\left(\mathrm{~m}, 4 \mathrm{H}, \mathrm{CH}_{\text {Ar-Bcat }}\right), 6.52\left(\mathrm{~s}, 2 \mathrm{H}, \mathrm{CH}_{\mathrm{Ar}}\right), 6.42\left(\mathrm{~s}, 2 \mathrm{H}, \mathrm{CH}_{\mathrm{Ar}}\right)$, 4.27 (br. s, $1 \mathrm{H}, \mathrm{BH}$ ), 3.45-3.15 (m, 12H, NCH$+\mathrm{CH}_{\text {iPr }}$ ), 2.26 (s, 6H, $\mathrm{CH}_{30-M e s}$ ), 2.12-2.11 (m, 9H, $\mathrm{CH}_{3 \mathrm{o} / \mathrm{p}-\mathrm{Mes}}$ ), 2.05 (s, 3H, $\left.\mathrm{CH}_{3 \mathrm{p}-\mathrm{Mes}}\right), 1.21-1.08$ (m, 24H, $\left.\mathrm{CH}_{3 \mathrm{Dip}}\right) \mathrm{ppm}$.

${ }^{13} \mathrm{C}\left\{{ }^{1} \mathrm{H}\right\} \mathrm{NMR}\left(125.8 \mathrm{MHz}, \mathrm{C}_{6} \mathrm{D}_{6}, 298 \mathrm{~K}\right): 194.5$ ( $C_{\text {Carbene, }}$, detected by HMBC), $192.4\left(C_{\text {Carbene }}\right.$ detected by HMBC), $151.0\left(C_{\mathrm{q}}\right), 147.4\left(C_{\mathrm{q}}\right), 146.8\left(C_{\mathrm{q}}\right), 140.1\left(C_{\mathrm{q}}\right), 137.6\left(C_{\mathrm{q}}\right), 137.2\left(C_{\mathrm{q}}\right)$, $137.1\left(C_{\mathrm{q}}\right), 136.4\left(C_{\mathrm{q}}\right), 136.0\left(C_{\mathrm{q}}\right), 135.8\left(C_{\mathrm{q}}\right), 135.3\left(C_{\mathrm{q}}\right), 129.8\left(C_{\mathrm{Ar}}\right), 129.3\left(\mathrm{CH}_{\mathrm{Ar}}\right), 128.6$ $\left(\mathrm{CH}_{\mathrm{Ar}}\right), 127.3\left(\mathrm{CH}_{\mathrm{Ar}}\right), 124.5\left(\mathrm{CH}_{\mathrm{Ar}}\right), 124.0\left(\mathrm{CH}_{\mathrm{Ar}}\right), 119.7\left(\mathrm{CH}_{\mathrm{Ar}, \mathrm{Bcat}}\right), 110.3\left(\mathrm{CH}_{\mathrm{Ar}, \mathrm{Bcat}}\right), 53.2$ $\left(\mathrm{NCH}_{2}\right), 52.5\left(\mathrm{NCH}_{2}\right), 50.2\left(\mathrm{NCH}_{2}\right), 49.2\left(\mathrm{NCH}_{2}\right), 28.6\left(\mathrm{CH}_{\mathrm{iPr}}\right), 28.3\left(\mathrm{CH}_{\mathrm{iPr}}\right), 26.4\left(\mathrm{CH}_{3 \mathrm{Dip}}\right), 25.5$ $\left(\mathrm{CH}_{3 \text { Dip }}\right), 24.4\left(\mathrm{CH}_{3 \text { Dip }}\right), 23.6\left(\mathrm{CH}_{3 \text { Dip }}\right), 21.2\left(\mathrm{CH}_{3 \text { Mes }}\right), 21.1\left(\mathrm{CH}_{3 \text { Mes }}\right), 19.3\left(\mathrm{CH}_{3 \text { Mes }}\right), 19.3$ $\left(\mathrm{CH}_{3 \mathrm{Mes}}\right) \mathrm{ppm}$.

${ }^{11} \mathrm{~B}$ NMR (160.5 MHz, $\left.\mathrm{C}_{6} \mathrm{D}_{6}, 298 \mathrm{~K}\right): 36.7$ (HBBBcat), 18.9 (HBBBcat) ppm. Note: The signal for B-Bcat could not be obtained due to broadening and overlapping with the signal at 36.7 ppm.

HRMS (LIFDI): calc.: $\left(\mathrm{C}_{54} \mathrm{H}_{69} \mathrm{~B}_{3} \mathrm{O}_{2} \mathrm{~N}_{4}+\mathrm{H}\right) \mathrm{m} / \mathrm{z}=839.5772$; found: $\mathrm{m} / \mathrm{z}=839.5742$.

UV/Vis: $\lambda_{\max (\text { pentane) }}: 579 \mathrm{~nm}$.

Synthesis of (SIDipMes)(Bcat)(H)B-B(H)(Bcat)(SIDipMes) (3)

HBcat $(8.4 \mathrm{mg}, 69.6 \mu \mathrm{mol})$ was added to a solution of $\mathrm{B}_{2}$ (SIDipMes) $)_{2}(20.0 \mathrm{mg}, 27.9 \mu \mathrm{mol})$ in benzene $(0.5 \mathrm{~mL})$. The solution turned from red to violet, then colorless crystals grew within minutes. These crystals were washed with benzene $(3 \times 0.5 \mathrm{~mL})$ and dried under reduced pressure. The product $\mathbf{3}$ was isolated in $82 \%$ yield as a colorless solid. Crystals suitable for $\mathrm{X}$-ray diffraction were obtained from the reaction mixture.

${ }^{1} \mathrm{H}\left\{{ }^{11} \mathrm{~B}\right\}$ NMR $\left(500.1 \mathrm{MHz}, \mathrm{CD}_{2} \mathrm{Cl}_{2}, 263 \mathrm{~K}\right): 7.18-7.15\left(\mathrm{~m}, 2 \mathrm{H}, \mathrm{CH}_{\mathrm{Ar}}\right), 7.06\left(\mathrm{~s}, 2 \mathrm{H}, \mathrm{CH}_{\mathrm{Ar}}\right)$, 6.97-6.96 (m, 4H, CH $\left.H_{\text {Ar,Bcat }}\right), 6.90-6.88\left(\mathrm{~m}, 4 \mathrm{H}, \mathrm{CH}_{\mathrm{Ar}, \mathrm{Bcat}}\right), 6.83\left(\mathrm{~s}, 2 \mathrm{H}, \mathrm{CH} \mathrm{Hr}_{\mathrm{Ar}}\right), 6.68(\mathrm{~s}, 2 \mathrm{H}$, $\left.\mathrm{CH}_{\mathrm{Ar}}\right), 6.13\left(\mathrm{~s}, 2 \mathrm{H}, \mathrm{CH}_{\mathrm{Ar}}\right), 4.10-3.97\left(\mathrm{~m}, 4 \mathrm{H}, \mathrm{NCH}_{2}\right), 3.77-3.70\left(\mathrm{~m}, 6 \mathrm{H}, \mathrm{NCH}_{2}+\mathrm{CH}_{\mathrm{iPr}}\right), 2.79$ (s,

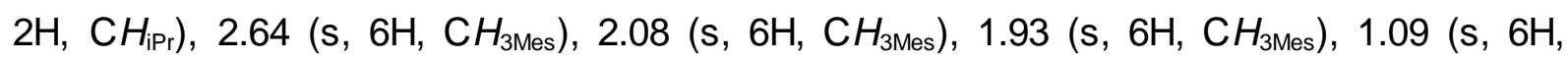


$\mathrm{CH}_{3 \text { Dip }}$ ), 0.86 (s, 6H, $\mathrm{CH}_{3 \mathrm{Dip}}$ ), 0.73 (s, 6H, $\left.\mathrm{CH}_{3 \text { Dip }}\right), 0.55$ (s, 2H, BH), -0,46 (s, 6H, $\mathrm{CH}_{3 \text { Dip }}$ ) ppm. Note: The signals at $7.35(\mathrm{~s}), 1.23(\mathrm{~m})$ and 0.89 (t) could be assigned to residual benzene and hexane.

${ }^{13} \mathrm{C}\left\{{ }^{1} \mathrm{H}\right\} \operatorname{NMR}\left(125.8 \mathrm{MHz}, \mathrm{CD}_{2} \mathrm{Cl}_{2}, 298 \mathrm{~K}\right): 150.4\left(C_{\mathrm{q}}\right), 147.4\left(C_{\mathrm{q}}\right), 147.3\left(C_{\mathrm{q}}\right), 138.7\left(C_{\mathrm{q}}\right)$, $136.9\left(C_{\mathrm{q}}\right), 136.7\left(C_{\mathrm{q}}\right), 129.6\left(\mathrm{CH}_{\mathrm{Ar}}\right), 128.5\left(\mathrm{CH}_{\mathrm{Ar}}\right), 127.4\left(\mathrm{CH}_{\mathrm{Ar}}\right), 124.2\left(\mathrm{CH}_{\mathrm{Ar}}\right), 123.6\left(\mathrm{CH}_{\mathrm{Ar}}\right)$, $120.4\left(\mathrm{CH}_{\text {ArBcat }}\right), 110.6\left(\mathrm{CH}_{\text {ArBcat }}\right), 52.6\left(\mathrm{NCH}_{2}\right), 51.1\left(\mathrm{NCH}_{2}\right), 28.3\left(\mathrm{CH}_{\mathrm{iPr}}\right), 27.3\left(\mathrm{CH}_{\mathrm{iPr}}\right), 26.7$ $\left(\mathrm{CH}_{3 \text { Dip }}\right), 25.4\left(\mathrm{CH}_{3 \text { Dip }}\right), 22.9\left(\mathrm{CH}_{3 \text { Dip }}\right), 21.6\left(\mathrm{CH}_{3 \text { Dip }}\right), 20.9\left(\mathrm{CH}_{3 \mathrm{Mes}}\right), 19.8\left(\mathrm{CH}_{3 \text { Mes }}\right), 19.4$ $\left(\mathrm{CH}_{3 \mathrm{Mes}}\right)$ ppm. Note: The signal at 128.68 could be assigned to residual benzene.

${ }^{11} \mathrm{~B}\left\{{ }^{1} \mathrm{H}\right\} \operatorname{NMR}\left(160.5 \mathrm{MHz}, \mathrm{CD}_{2} \mathrm{Cl}_{2}, 298 \mathrm{~K}\right): 45.2$ (BBcat), -33.8 (BBH) ppm.

$\mathbb{R}: \tilde{v}(\mathrm{BH}): 2366 \mathrm{~cm}^{-1}$.

HRMS (LIFDI): calc. $\left(\mathrm{C}_{60} \mathrm{H}_{74} \mathrm{~B}_{4} \mathrm{O}_{4} \mathrm{~N}_{4}\right) \mathrm{m} / \mathrm{z}=958.6077$; found: $\mathrm{m} / \mathrm{z}=958.6048$.

\section{Synthesis of [(SIDipMes)(Bcat)B-B(H)(Bcat)(SIDipMes)][OTf] (4)}

Compound 2 (20 mg, $20.8 \mu \mathrm{mol})$ were suspended in $\mathrm{CH}_{2} \mathrm{Cl}_{2}(10 \mathrm{~mL})$ and treated with MeOTf $(5.0 \mathrm{mg}, 29.5 \mu \mathrm{mol})$. Immediate gas formation was observed. After ${ }^{11} \mathrm{~B}$ NMR screening, no starting material remained. All volatiles were removed in vacuo and benzene $(0.5 \mathrm{~mL})$ was added to the resulting oily residue. Placing the mixture in an ultrasound bath for $30 \mathrm{~min}$ resulted in precipitation of a colorless solid, which was washed with benzene $(3 \times 0.5 \mathrm{~mL})$ and dried in vacuo. The product $\mathbf{4}$ was isolated in $83 \%$ yield as a colorless solid. Crystals of 4a suitable for X-ray diffraction were obtained by diffusion of pentane into a saturated solution of $\mathbf{4}$ in $\mathrm{CH}_{2} \mathrm{Cl}_{2}$.

Two isomers (4a, major/4b, minor): $1 / 0.75$. If exchange were occurring between $\mathbf{4 a}$ and $\mathbf{4 b}$, we would have expected to observe exchange peaks in the NOESY and ROESY spectra of the compound mixture. As this was not the case, we conclude that exchange is either not taking place or is extremely slow.

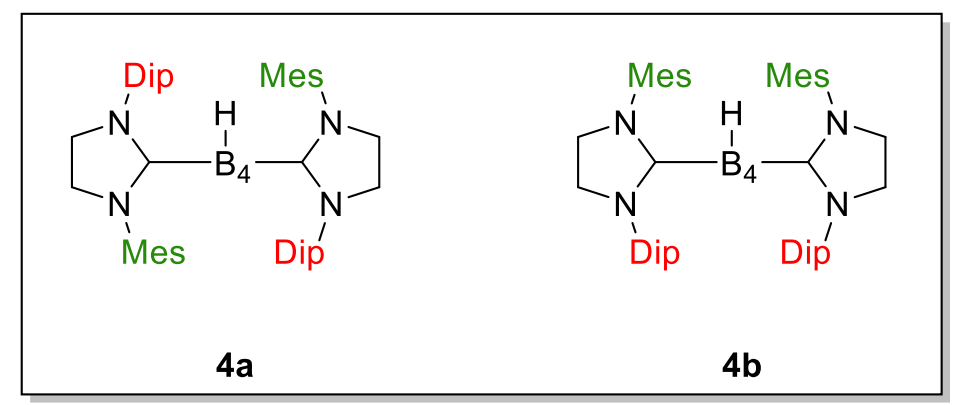


Characteristic signals for the major isomer (4a): ${ }^{1} \mathrm{H} \mathrm{NMR}\left(500.1 \mathrm{MHz}, \mathrm{CD}_{2} \mathrm{Cl}_{2}, 288 \mathrm{~K}\right): 7.40$ (m, 2H, CH $\left.H_{\text {Ar,p-Dip }}\right), 7.25-7.22\left(\mathrm{~m}, 6 \mathrm{H}, \mathrm{CH}_{\text {Ar }}+\mathrm{CH}_{\text {cat }}\right), 7.20-7.16\left(\mathrm{~m}, 6 \mathrm{H}, \mathrm{CH}_{\text {Ar }}+\mathrm{CH}_{\text {cat }}\right), 7.02$ - $7.00\left(\mathrm{~m}, 2 \mathrm{H}, \mathrm{CH}_{\mathrm{Ar}}\right), 6.92-6.90\left(\mathrm{~m}, 2 \mathrm{H}, \mathrm{CH}_{\mathrm{Ar}}\right), 6.38\left(\mathrm{~m}, 2 \mathrm{H}, \mathrm{CH}_{\mathrm{Ar}}\right), 4.46-4.40(\mathrm{~m}, 2 \mathrm{H}$, $\mathrm{NCH}_{2}$ ), $4.16-3.98\left(\mathrm{~m}, 6 \mathrm{H}, \mathrm{NCH}_{2}\right.$ ), 3.70 (sept. $2 \mathrm{H},{ }^{3} \mathrm{~J}_{\mathrm{H}, \mathrm{H}}=6.77 \mathrm{~Hz}, \mathrm{CH}_{\mathrm{iPr}}$ ), 3.34 (sept. $2 \mathrm{H}$, $\left.{ }^{3} J_{\mathrm{H}, \mathrm{H}}=6.77 \mathrm{~Hz}, \mathrm{CH}_{\mathrm{iPr}}\right), 2.59\left(\mathrm{~s}, 6 \mathrm{H}, \mathrm{CH}_{3 \mathrm{Mes}}\right), 2.28\left(\mathrm{~s}, 6 \mathrm{H}, \mathrm{CH}_{3 \mathrm{Mes}}\right), 2.16\left(\mathrm{~m}, 6 \mathrm{H}, \mathrm{CH}_{3 \text { Mes }}\right)$, $1.11\left(\mathrm{~d}, 12 \mathrm{H},{ }^{3} \mathrm{~J}_{\mathrm{H}, \mathrm{H}}=6.77 \mathrm{~Hz}, \mathrm{CH}_{3 \mathrm{Dip}}\right), 1.09\left(\mathrm{~d}, 12 \mathrm{H},{ }^{3} \mathrm{~J}_{\mathrm{H}, \mathrm{H}}=6.77 \mathrm{~Hz}, \mathrm{CH}_{3 \mathrm{Dip}}\right), 0.77(\mathrm{~d}, 12 \mathrm{H}$, $\left.{ }^{3} J_{\mathrm{H}, \mathrm{H}}=6.77 \mathrm{~Hz}, \mathrm{CH} H_{3 \mathrm{Dip}}\right), 0.33\left(\mathrm{~d}, 12 \mathrm{H},{ }^{3} J_{\mathrm{H}, \mathrm{H}}=6.77 \mathrm{~Hz}, \mathrm{C} H_{3 \mathrm{Dip}}\right),-2.39(\mathrm{BHB}) \mathrm{ppm}$.

Characteristic signals for the minor isomer (4b): ${ }^{1} \mathrm{H} \mathrm{NMR}\left(500.1 \mathrm{MHz}, \mathrm{CD}_{2} \mathrm{Cl}_{2}, 263 \mathrm{~K}\right): 3.56$ (sept. $2 \mathrm{H},{ }^{3} \mathrm{~J}_{\mathrm{H}, \mathrm{H}}=6.77 \mathrm{~Hz}, \mathrm{CH}_{\mathrm{iPr}}$ ), 3.46 (sept. $2 \mathrm{H},{ }^{3} \mathrm{~J}_{\mathrm{H}, \mathrm{H}}=6.77 \mathrm{~Hz}, \mathrm{CH}_{\mathrm{iPr}}$ ), 2.76 (s, 3H, CH $\mathrm{H}_{3 \mathrm{Mes}}$ )

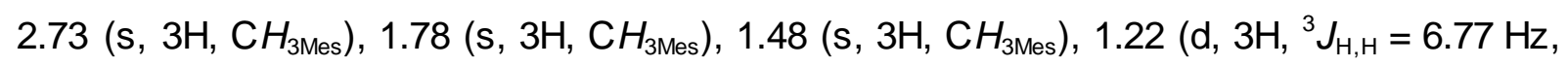
$\left.\mathrm{CH}_{3 \mathrm{Dip}}\right), 1.00\left(\mathrm{~s}, 6 \mathrm{H}, \mathrm{CH}_{3 \mathrm{Mes}}\right), 0.93\left(\mathrm{~d}, 3 \mathrm{H},{ }^{3} \mathrm{~J}_{\mathrm{H}, \mathrm{H}}=6.77 \mathrm{~Hz}, \mathrm{CH}_{3 \mathrm{Dip}}\right), 0.80\left(\mathrm{~d}, 6 \mathrm{H},{ }^{3} J_{\mathrm{H}, \mathrm{H}}=6.77\right.$ $\left.\mathrm{Hz}, \mathrm{CH}_{3 \mathrm{Dip}}\right),-0.42\left(\mathrm{~d}, 6 \mathrm{H},{ }^{3} \mathrm{~J}_{\mathrm{H}, \mathrm{H}}=6.77 \mathrm{~Hz}, \mathrm{CH}_{3 \mathrm{Dip}}\right),-0.58\left(\mathrm{~d}, 6 \mathrm{H},{ }^{3} J_{\mathrm{H}, \mathrm{H}}=6.77 \mathrm{~Hz}, \mathrm{CH}_{3 \text { Dip }}\right),-$ 2.32 (BHB) ppm, note: the aromatic signals could not be separated due to overlap of the signals of the isomers.

(A): ${ }^{13} \mathrm{C}\left\{{ }^{1} \mathrm{H}\right\} \operatorname{NMR}\left(125.8 \mathrm{MHz}, \mathrm{CD}_{2} \mathrm{Cl}_{2}, 263 \mathrm{~K}\right): 188.5$ ( $\left.C_{\text {Carbene }}\right), 188.1\left(C_{\text {Carbene }}\right), 148.1\left(C_{\mathrm{q}}\right)$, $147.7\left(C_{\mathrm{q}}\right), 145.2\left(C_{\mathrm{q}}\right), 138.9\left(C_{\mathrm{q}}\right), 138.8\left(C_{\mathrm{q}}\right), 138.5\left(C_{\mathrm{q}}\right), 136.7\left(C_{\mathrm{q}}\right), 135.3\left(C_{\mathrm{q}}\right)$, $134.5\left(C_{\mathrm{q}}\right), 134.0\left(C_{\mathrm{q}}\right), 133.6\left(C_{\mathrm{q}}\right), 132.5\left(C_{\mathrm{q}}\right), 132.0\left(C_{\mathrm{q}}\right), 130.3\left(\mathrm{CH}_{\mathrm{Ar}}\right), 130.2\left(\mathrm{CH}_{\mathrm{Ar}}\right), 130.1$ $\left(\mathrm{CH}_{\mathrm{Ar}}\right), 129.7\left(\mathrm{CH}_{\mathrm{Ar}}\right), 125.3\left(\mathrm{CH}_{\mathrm{Ar}}\right), 125.0\left(\mathrm{CH}_{\mathrm{Ar}}\right), 123.0\left(\mathrm{CH}_{\mathrm{Ar}}\right), 112.0\left(\mathrm{CH}_{\mathrm{Ar}}\right), 54.0\left(\mathrm{NCH}_{2}\right)$, $51.8\left(\mathrm{NCH}_{2}\right), 28.9\left(\mathrm{CH}_{\mathrm{iPr}}\right), 27.4\left(\mathrm{CH}_{\mathrm{iPr}}\right), 27.0\left(\mathrm{CH}_{3 \mathrm{Dip}}\right), 25.7\left(\mathrm{CH}_{3 \mathrm{Dip}}\right), 23.6\left(\mathrm{CH}_{3 \mathrm{Dip}}\right), 22.0$ $\left(\mathrm{CH}_{3 \mathrm{Dip}}\right), 21.0\left(\mathrm{CH}_{3 \mathrm{Mes}}\right), 20.8\left(\mathrm{CH}_{3 \mathrm{Mes}}\right), 20.7\left(\mathrm{CH}_{3 \mathrm{Mes}}\right), 18.9\left(\mathrm{CH}_{3 \mathrm{Mes}}\right), 16.8\left(\mathrm{CH}_{3 \mathrm{Mes}}\right) \mathrm{ppm}$.

(B) ${ }^{13} \mathrm{C}\left\{{ }^{1} \mathrm{H}\right\} \mathrm{NMR}\left(125.8 \mathrm{MHz}, \mathrm{CD}_{2} \mathrm{Cl}_{2}, 263 \mathrm{~K}\right): 189.5\left(C_{\text {Carbene }}\right), 148.1\left(C_{\mathrm{q}}\right), 148.0\left(C_{\mathrm{q}}\right), 147.6$ $\left(C_{\mathrm{q}}\right), 147.2\left(C_{\mathrm{q}}\right), 146.6\left(C_{\mathrm{q}}\right), 145.5\left(C_{\mathrm{q}}\right), 136.8\left(C_{\mathrm{q}}\right), 136.7\left(C_{\mathrm{q}}\right), 136.4\left(C_{\mathrm{q}}\right), 136.0\left(C_{\mathrm{q}}\right), 132.1$ $\left(C_{\mathrm{q}}\right), 130.5\left(\mathrm{CH}_{\mathrm{Ar}}\right), 130.4\left(\mathrm{CH}_{\mathrm{Ar}}\right), 130.0\left(\mathrm{CH}_{\mathrm{Ar}}\right), 129.6\left(\mathrm{CH}_{\mathrm{Ar}}\right), 128.5\left(\mathrm{CH}_{\mathrm{Ar}}\right), 125.5\left(\mathrm{CH}_{\mathrm{Ar}}\right)$, $125.3\left(\mathrm{CH}_{\mathrm{Ar}}\right), 124.8\left(\mathrm{CH}_{\mathrm{Ar}}\right), 124.6\left(\mathrm{CH}_{\mathrm{Ar}}\right), 123.7\left(\mathrm{CH}_{\mathrm{Ar}}\right), 112.4\left(\mathrm{CH}_{\mathrm{Ar}}\right), 54.2\left(\mathrm{NCH}_{2}\right), 51.7$ $\left(\mathrm{NCH}_{2}\right), 29.0\left(\mathrm{CH}_{\mathrm{iPr}}\right), 28.9\left(\mathrm{CH}_{\mathrm{iPr}}\right), 27.5\left(\mathrm{CH}_{3 \mathrm{Dip}}\right), 27.2\left(\mathrm{CH}_{3 \text { Dip }}\right), 26.3\left(\mathrm{CH}_{3 \mathrm{Dip}}\right), 25.6\left(\mathrm{CH}_{3 \mathrm{Dip}}\right)$, $23.1\left(\mathrm{CH}_{3 \mathrm{Dip}}\right), 22.2\left(\mathrm{CH}_{3 \mathrm{Dip}}\right), 20.1\left(\mathrm{CH}_{3 \mathrm{Mes}}\right), 19.6\left(\mathrm{CH}_{3 \text { Mes }}\right), 19.1,19.0\left(\mathrm{CH}_{3 \mathrm{Mes}}\right) \mathrm{ppm}$.

note: the signals at $128.1,127.9,127.7$ can be assigned to residual $C_{6} D_{6}$, the carbon atom of the $\mathrm{CF}_{3}$-group can be found at $121.0\left(q,{ }^{1} \mathrm{~J}_{13 \mathrm{C}, 19 \mathrm{~F}}=320 \mathrm{~Hz}\right)$.

${ }^{11} \mathrm{~B}$ NMR (160.5 MHz, $\mathrm{CD}_{2} \mathrm{Cl}_{2}, 308 \mathrm{~K}$ ): 35.9 (B-Bcat, calc (B3LYP-611G(d)) 34.4), 23.6 (HB-Bcat, calc (B3LYP-611G(d)) 20.8) ppm.

${ }^{19} \mathrm{~F}\left\{{ }^{1} \mathrm{H}\right\} \mathrm{NMR}\left(376.5 \mathrm{MHz}, \mathrm{CD}_{2} \mathrm{Cl}_{2}, 298 \mathrm{~K}\right):-79.0 \mathrm{ppm}$

HRMS (LIFDI): calc. $\left(\mathrm{C}_{60} \mathrm{H}_{73} \mathrm{~B}_{4} \mathrm{O}_{4} \mathrm{~N}_{4}\right) \mathrm{m} / \mathrm{z}=957.5999$; found: $\mathrm{m} / \mathrm{z}=957.5980$. 


\section{NMR Spectra}

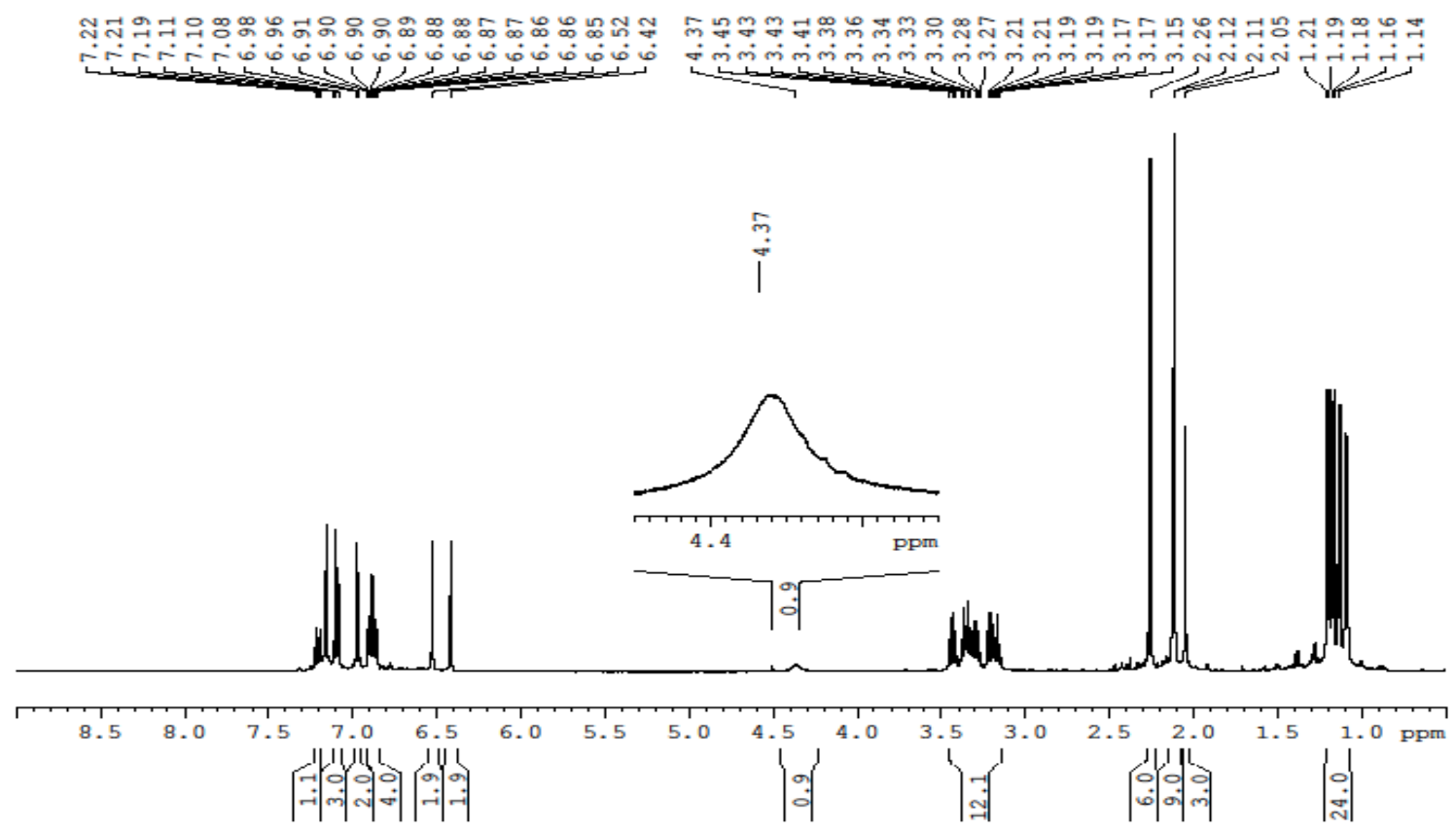

Figure $\mathrm{S} 1{ }^{1} \mathrm{H}\left\{{ }^{11} \mathrm{~B}\right\}$ NMR spectrum of 2 


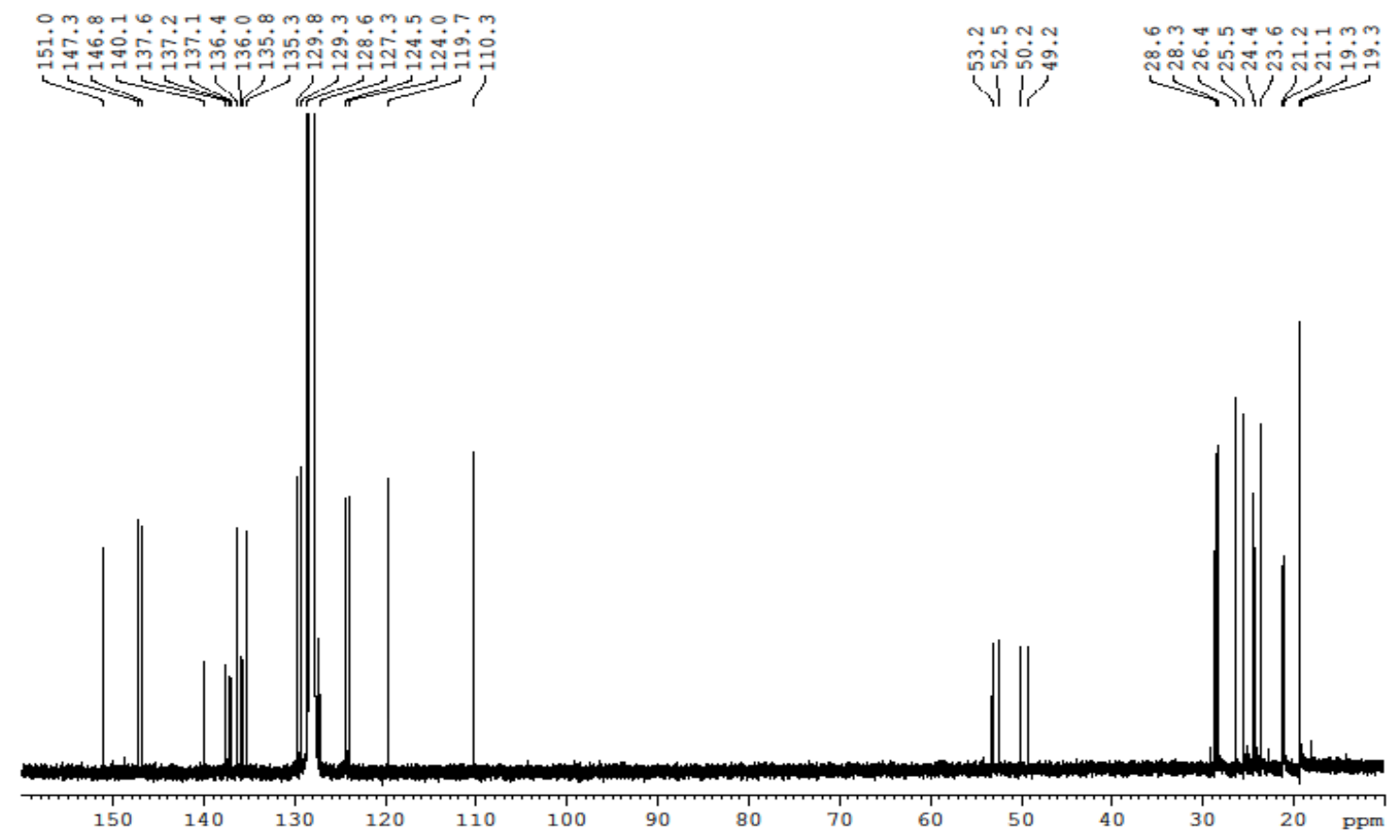

Figure $\mathbf{S} 2{ }^{13} \mathrm{C}\left\{{ }^{1} \mathrm{H}\right\}$ NMR spectrum of $\mathbf{2}$

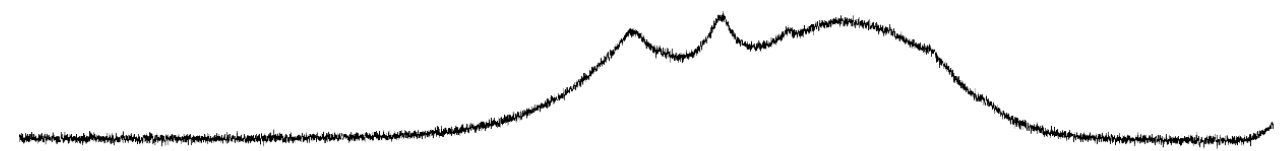

Figure $\mathrm{S} 3{ }^{11} \mathrm{~B}$ NMR spectrum of 2 


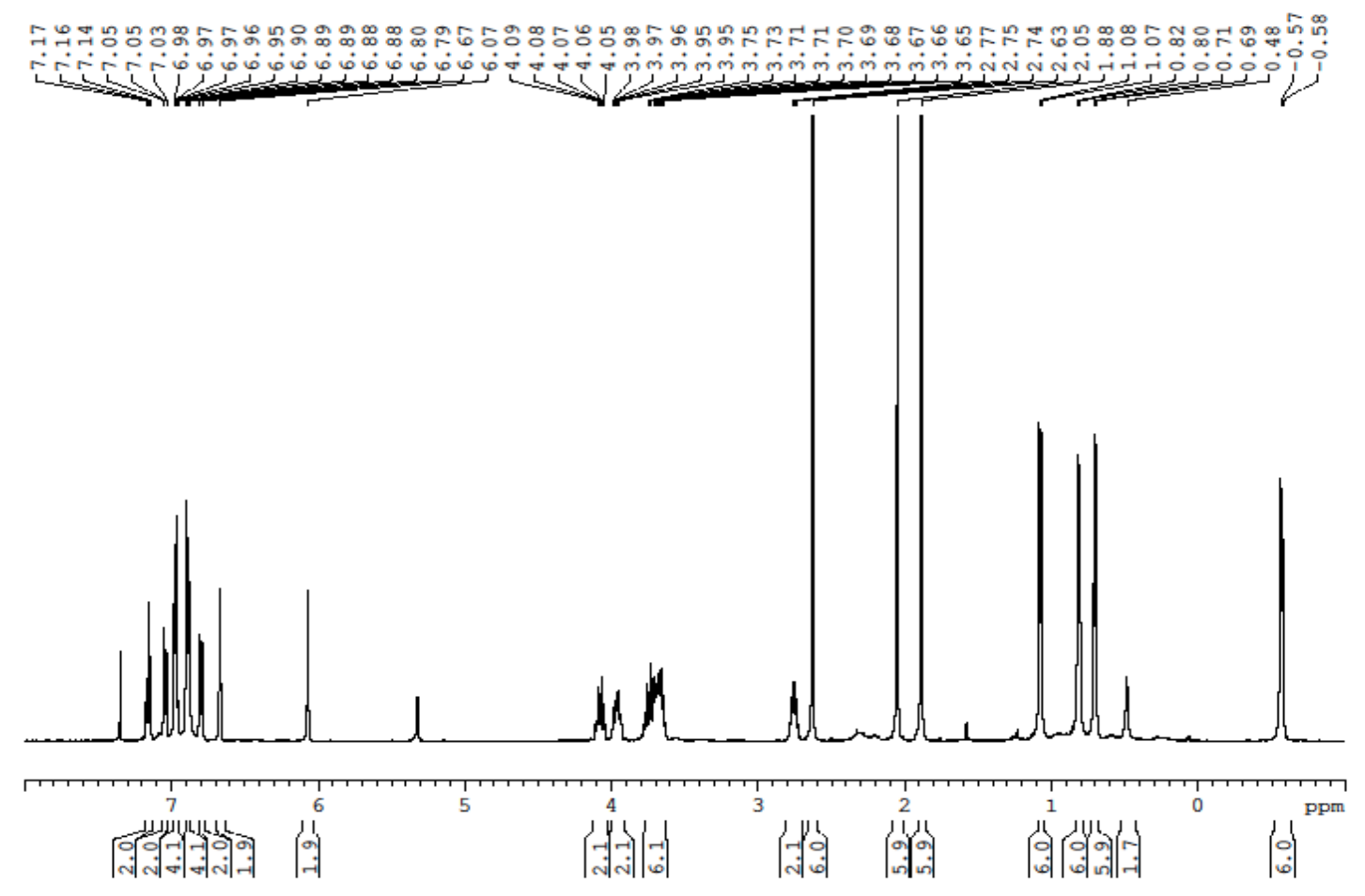

Figure S4 ${ }^{1} \mathrm{H}\left\{{ }^{11} \mathrm{~B}\right\}$ NMR spectrum of $\mathbf{3}$

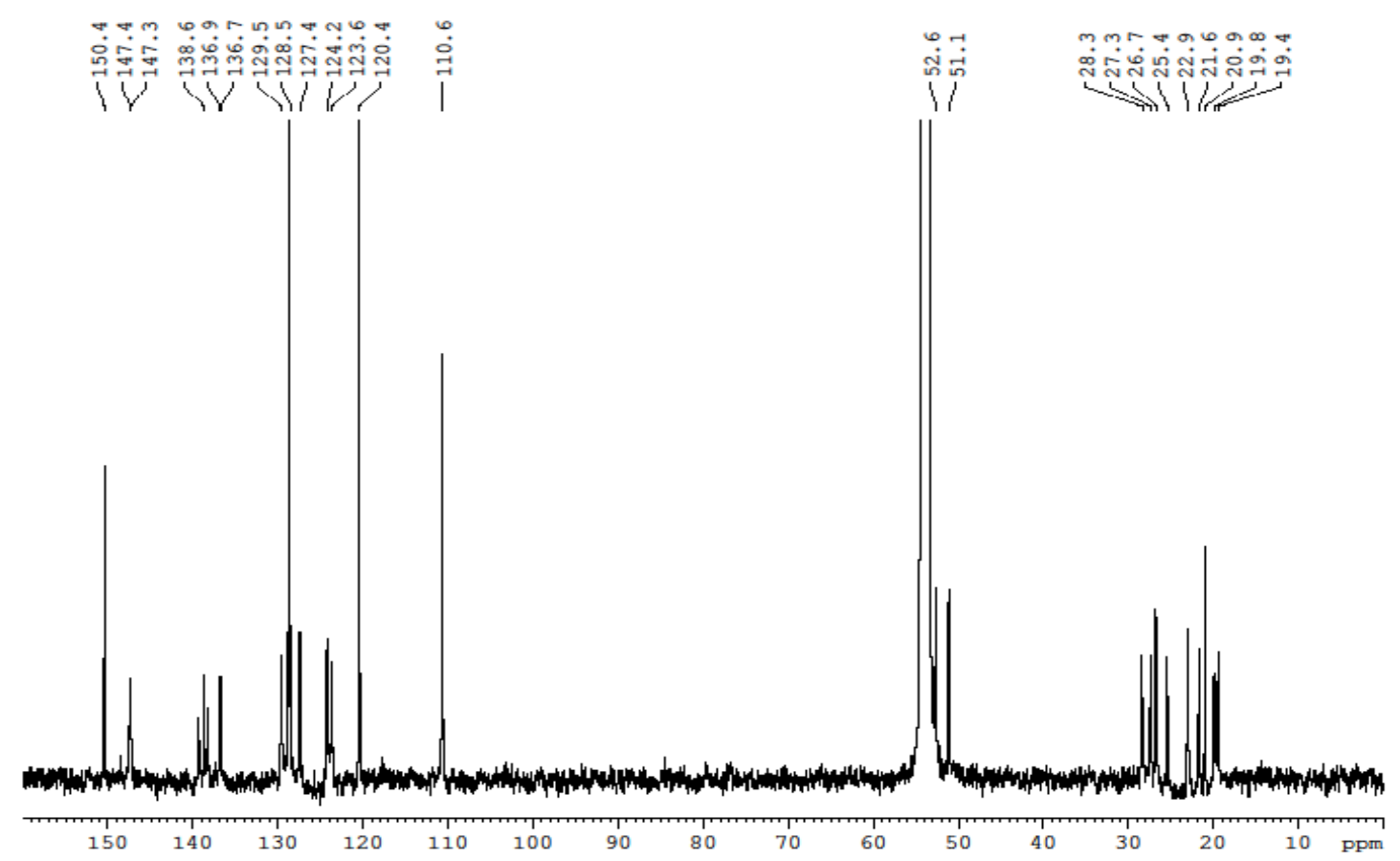

Figure $\mathbf{S} 5{ }^{13} \mathrm{C}\left\{{ }^{1} \mathrm{H}\right\}$ NMR spectrum of $\mathbf{3}$ 


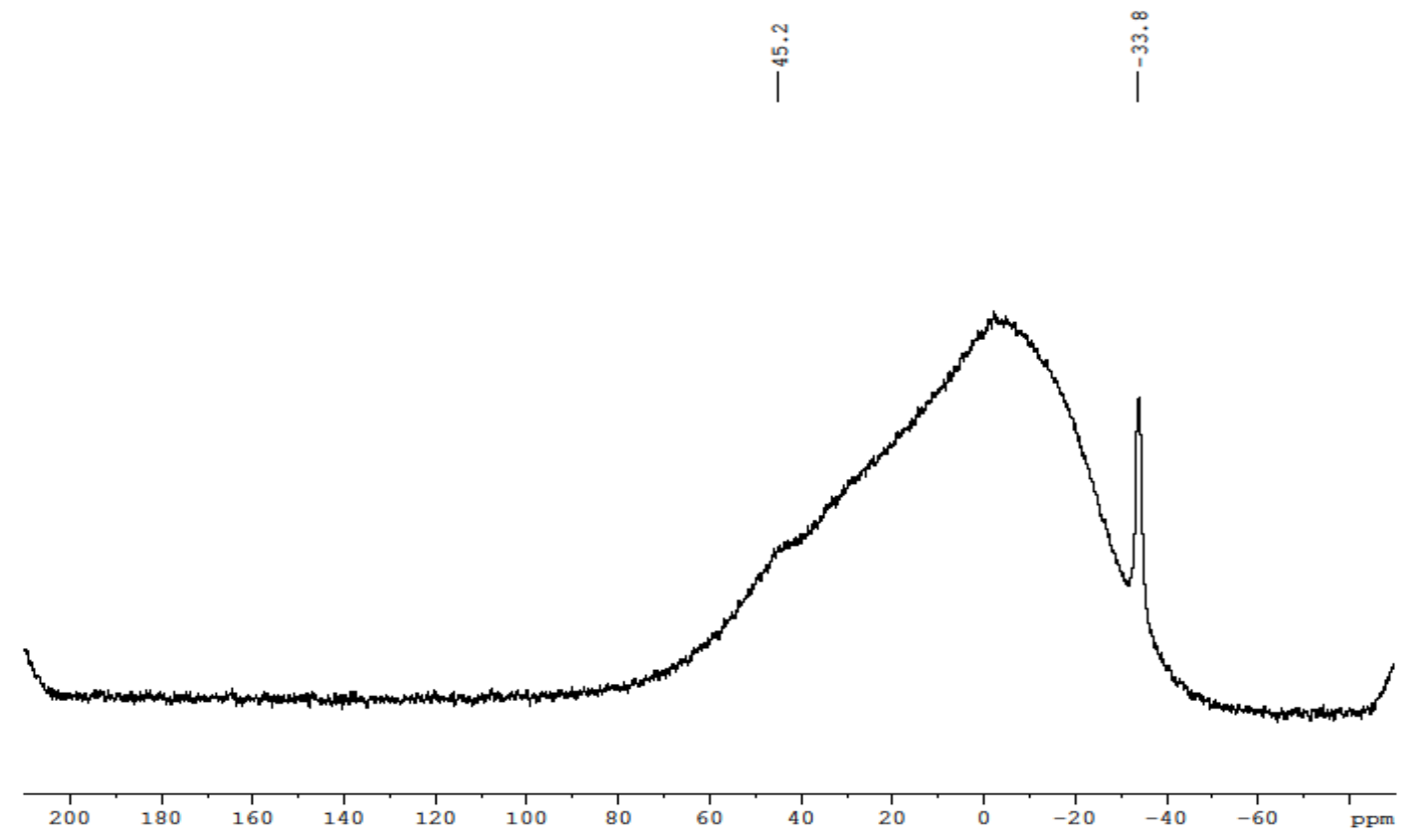

Figure $\mathbf{S} 6{ }^{11} \mathrm{~B}\left\{{ }^{1} \mathrm{H}\right\}$ NMR spectrum of 3

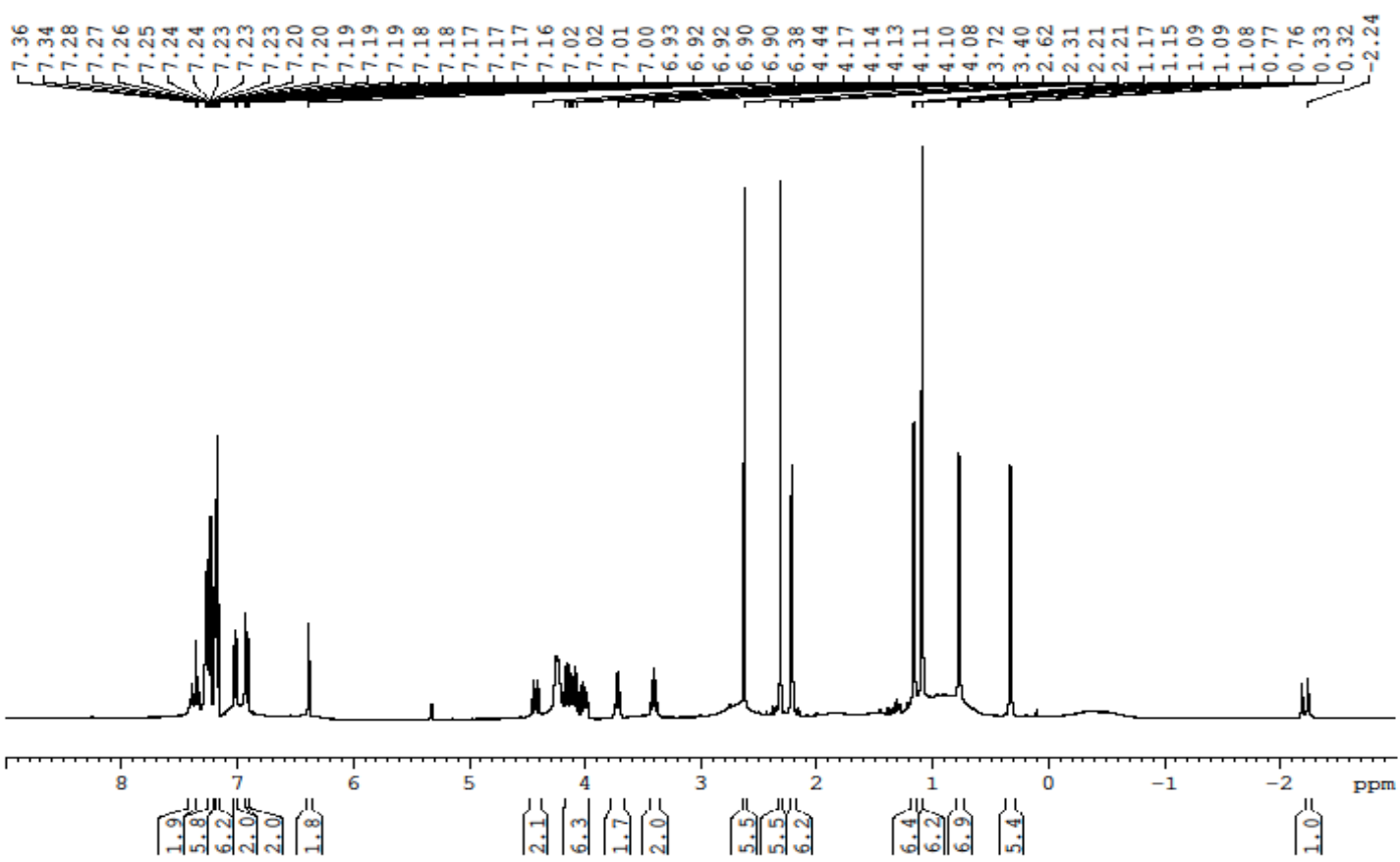


Figure $\mathbf{S} 7{ }^{1} \mathrm{H}\left\{{ }^{11} \mathrm{~B}\right\}$ NMR spectrum of $4(298 \mathrm{~K}$, both isomers, minor isomer appears as broad signals)

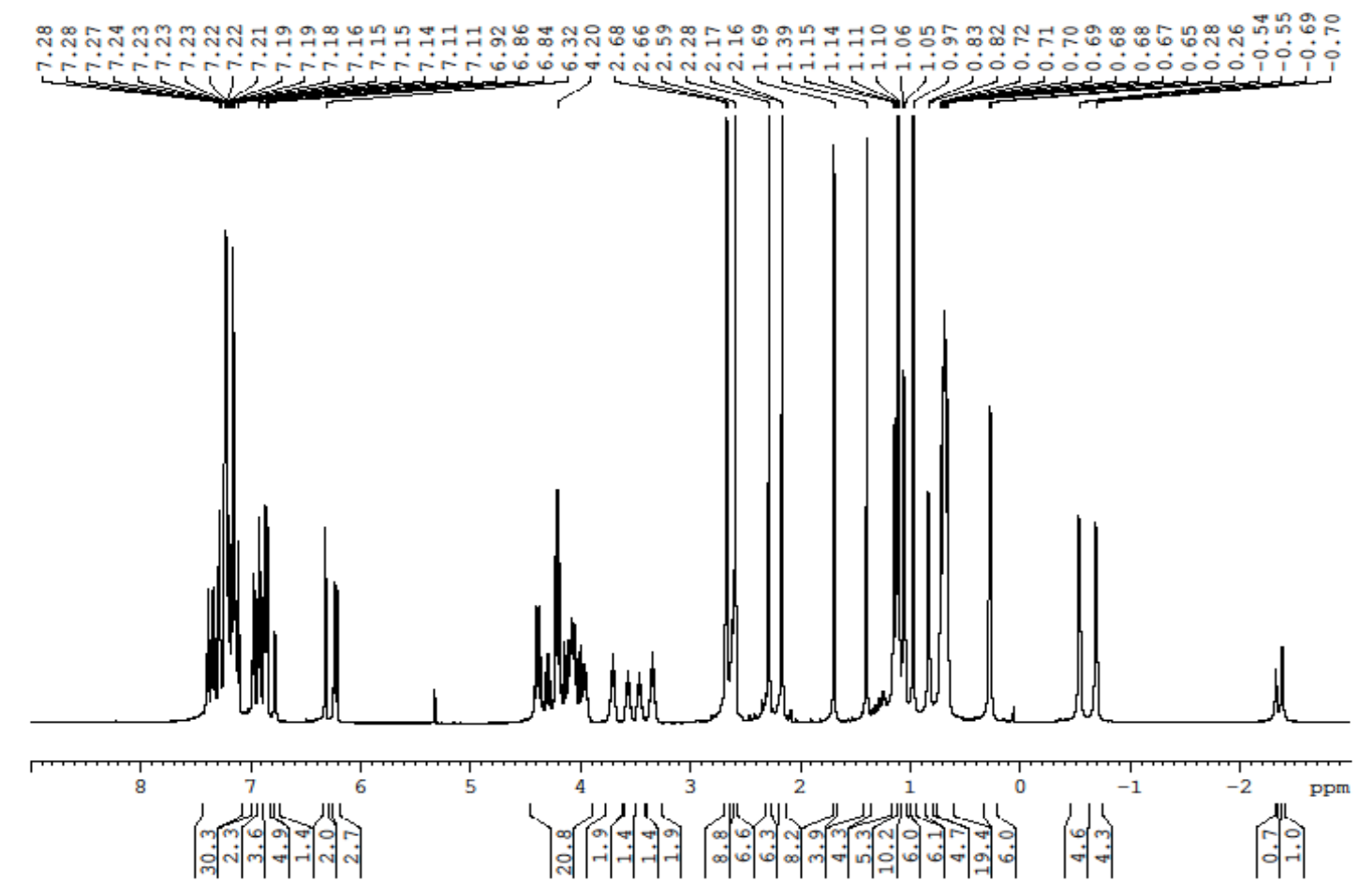

Figure S8 ${ }^{1} H\left\{{ }^{11} \mathrm{~B}\right\}$ NMR spectrum of $\mathbf{4}$ (263 K, both isomers appear as sharp signals) 


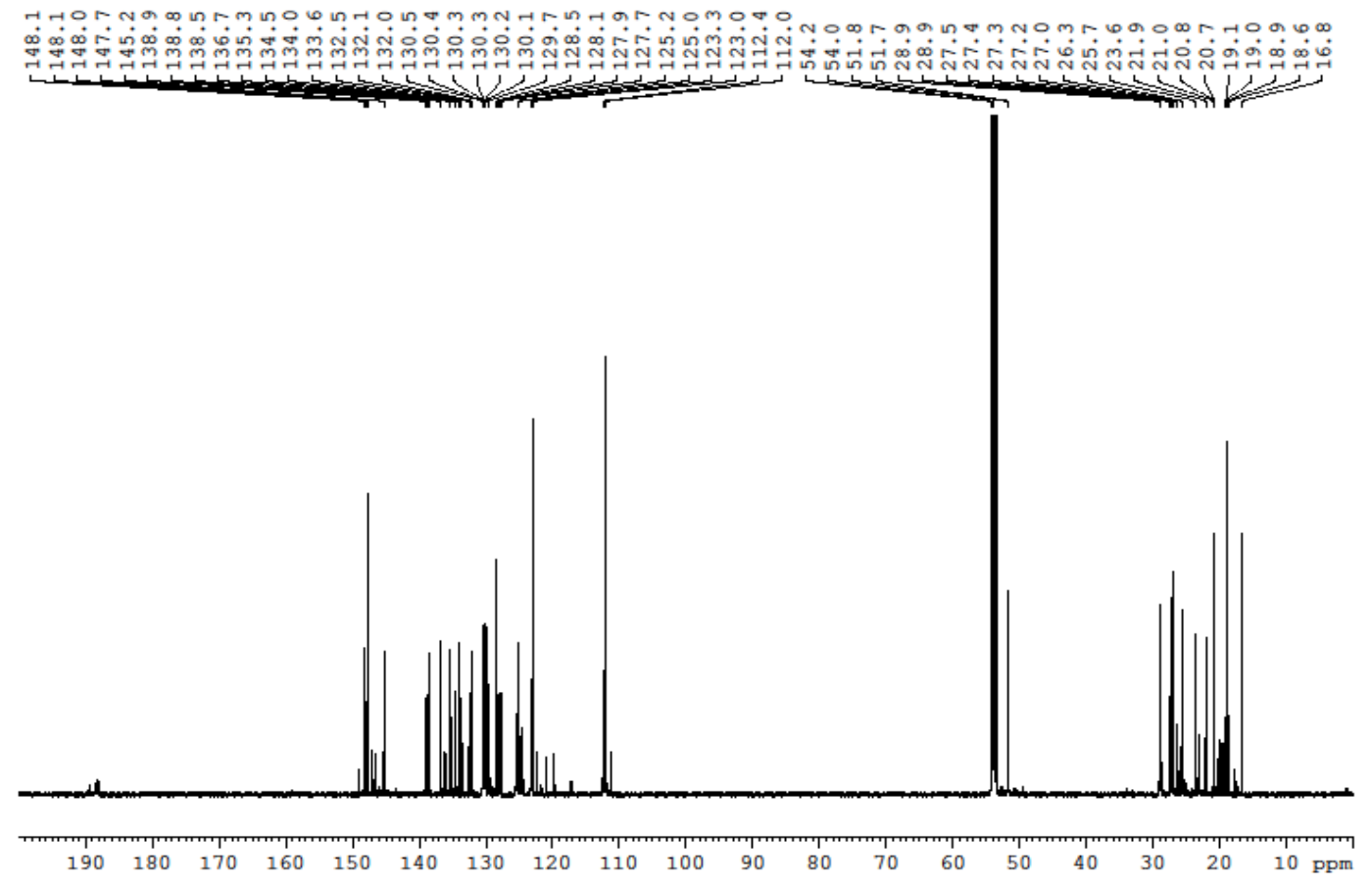

Figure $S 9{ }^{13} \mathrm{C}\left\{{ }^{1} \mathrm{H}\right\}$ NMR spectrum of $4(263 \mathrm{~K})$

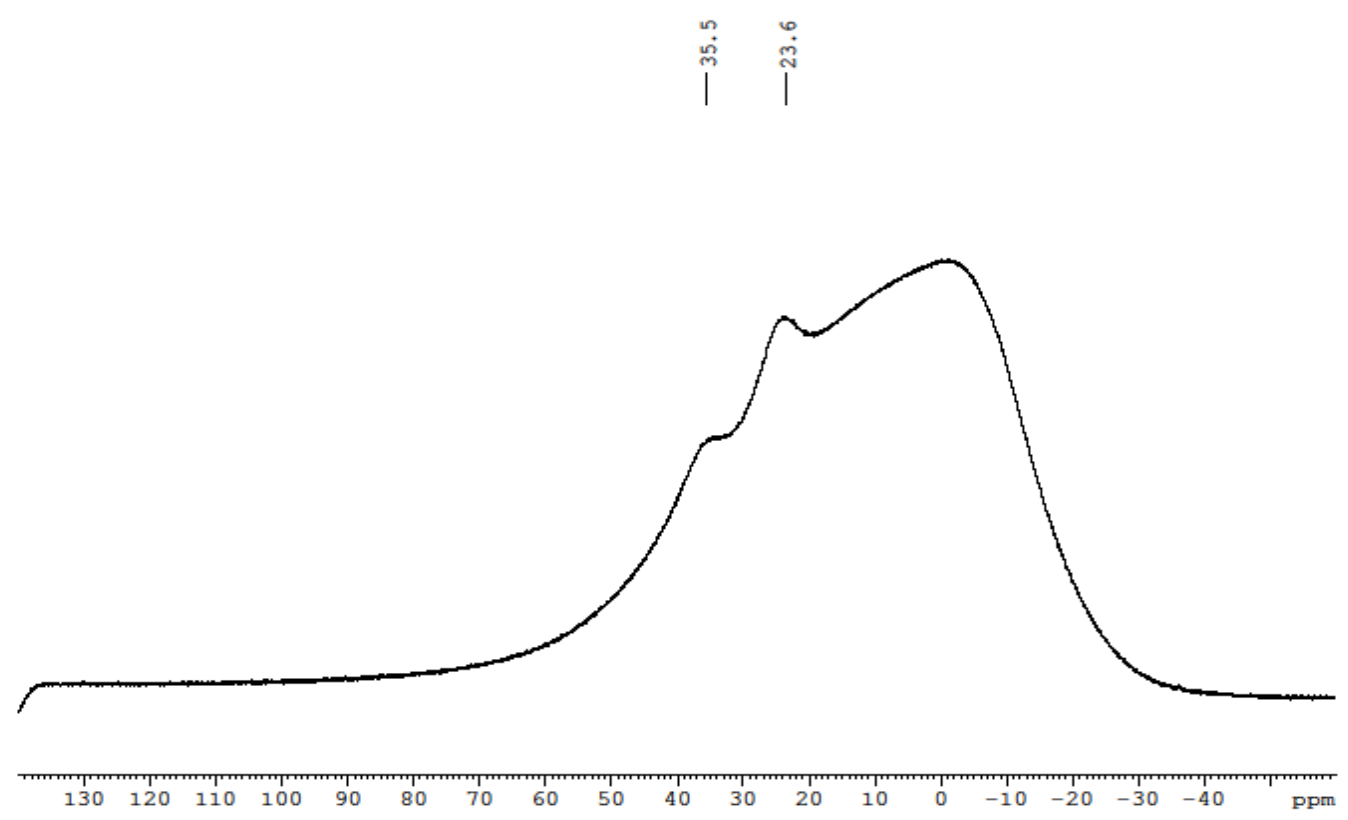

Figure $\mathrm{S} 10^{11} \mathrm{~B}\left\{{ }^{1} \mathrm{H}\right\}$ NMR spectrum of $4(308 \mathrm{~K})$ 


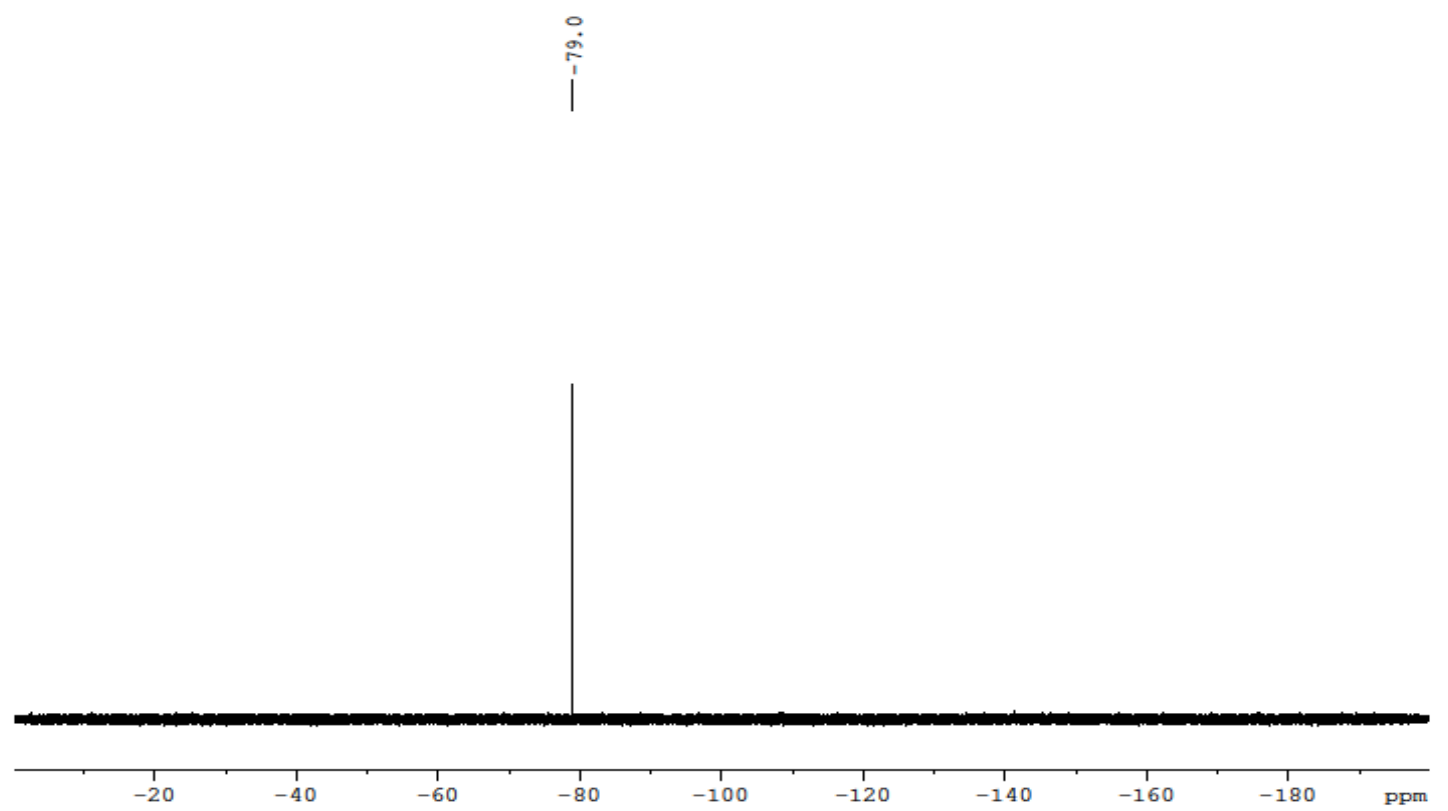

Figure $\mathrm{S} 11{ }^{19} \mathrm{~F}$ NMR spectrum of 4 (298 K) 


\section{IR Spectra}

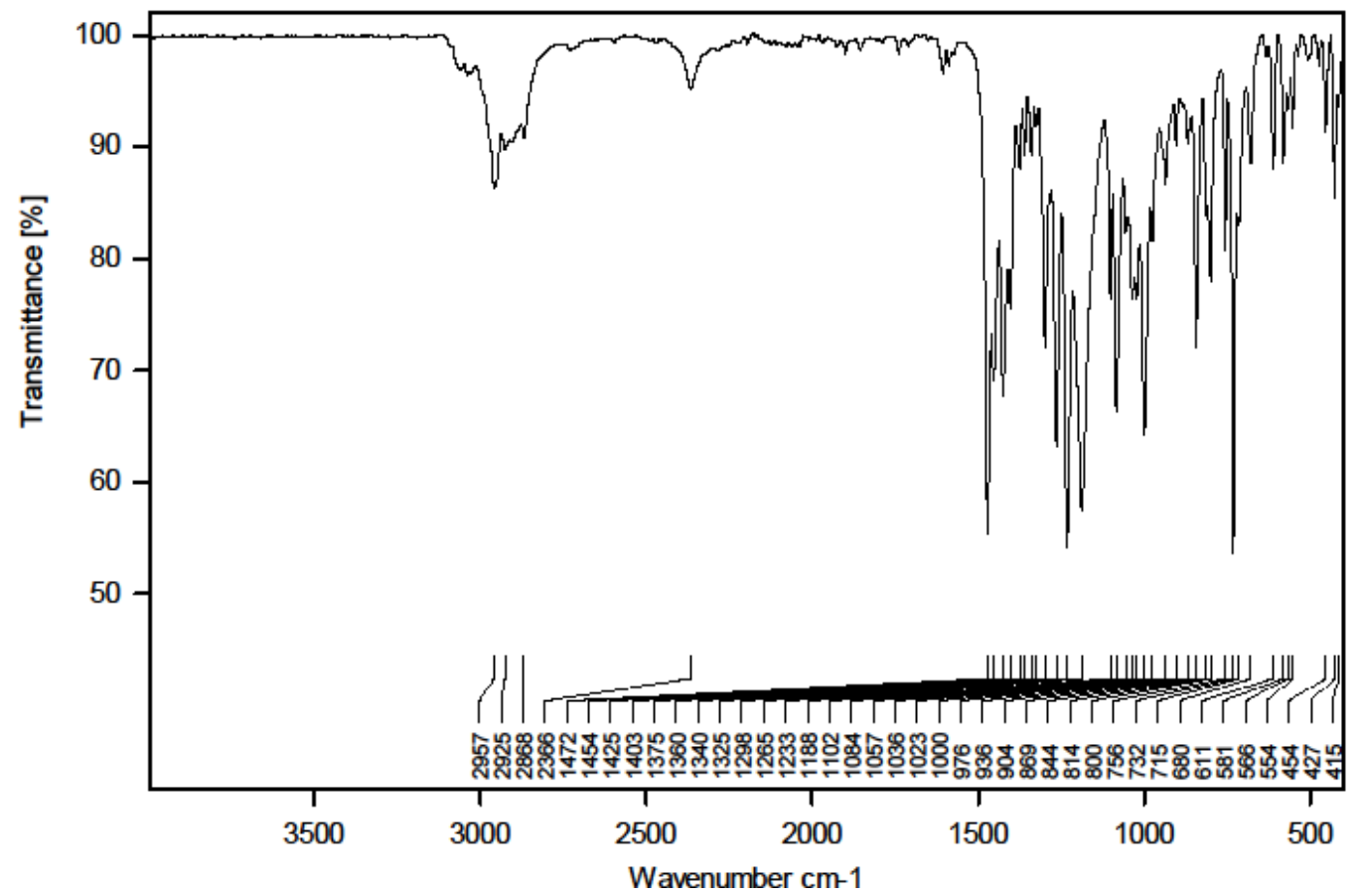

Figure S12 IR spectrum of $\mathbf{3}$ in the solid state (298 K)

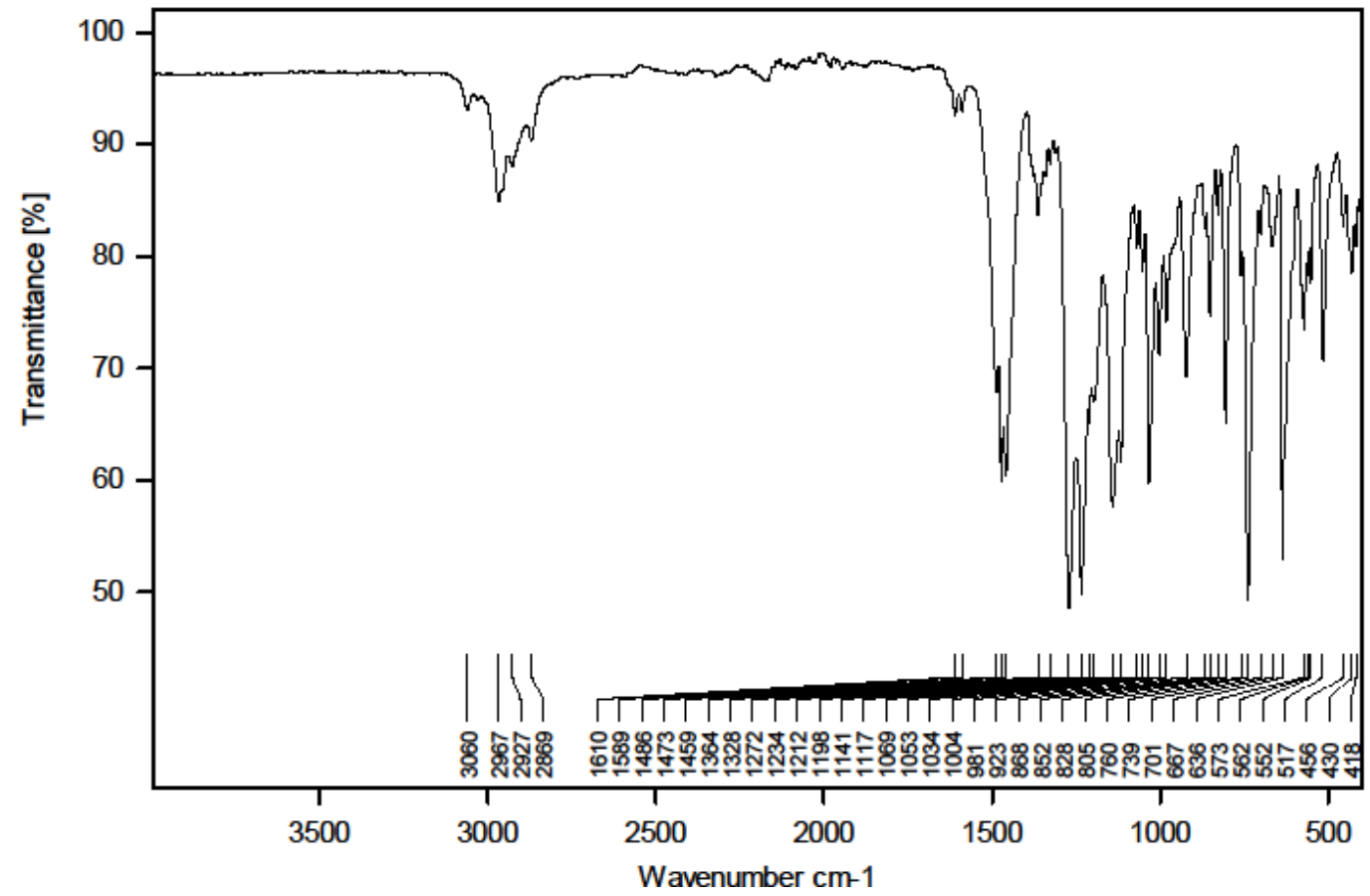

Figure S13 IR spectrum of 4 in the solid state (298 K) 


\section{UV-Vis spectra}

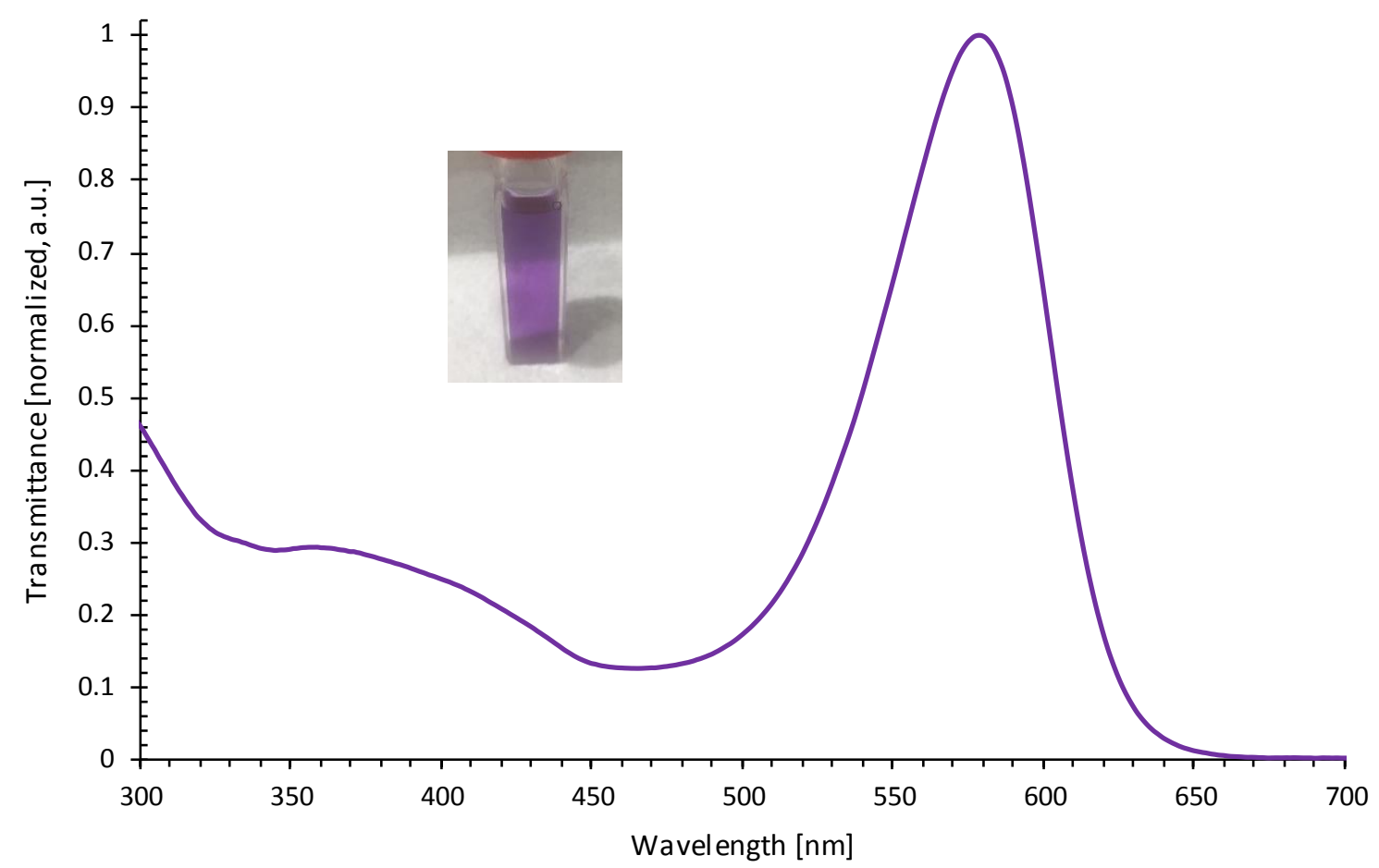

Figure S14 UV/Vis spectrum of 4 in pentane (298 K)

\section{Computational Details}

Initially, we performed geometry optimization calculations for 2, 3 and $\mathbf{4}$ starting from the experimental crystallographic data. The structures were optimized using density functional theory (DFT) at the B3LYP/6-311G(d) level with the Gaussian 09 program package. ${ }^{4}$ The optimized structures were only slightly different than those derived from their respective $X$ ray diffraction data. All geometries were characterized as minimum energy structures in their respective potential energy surfaces, as all vibrational frequencies were found to be real. The calculations of the natural population analysis (NPA) and the Wiberg bond indices (WBls) were performed with the NBO 6 program package. ${ }^{5}$ The visualisation of the molecular orbitals was performed with the open-source molecular builder and visualization tool Avogadro. ${ }^{6}$ 

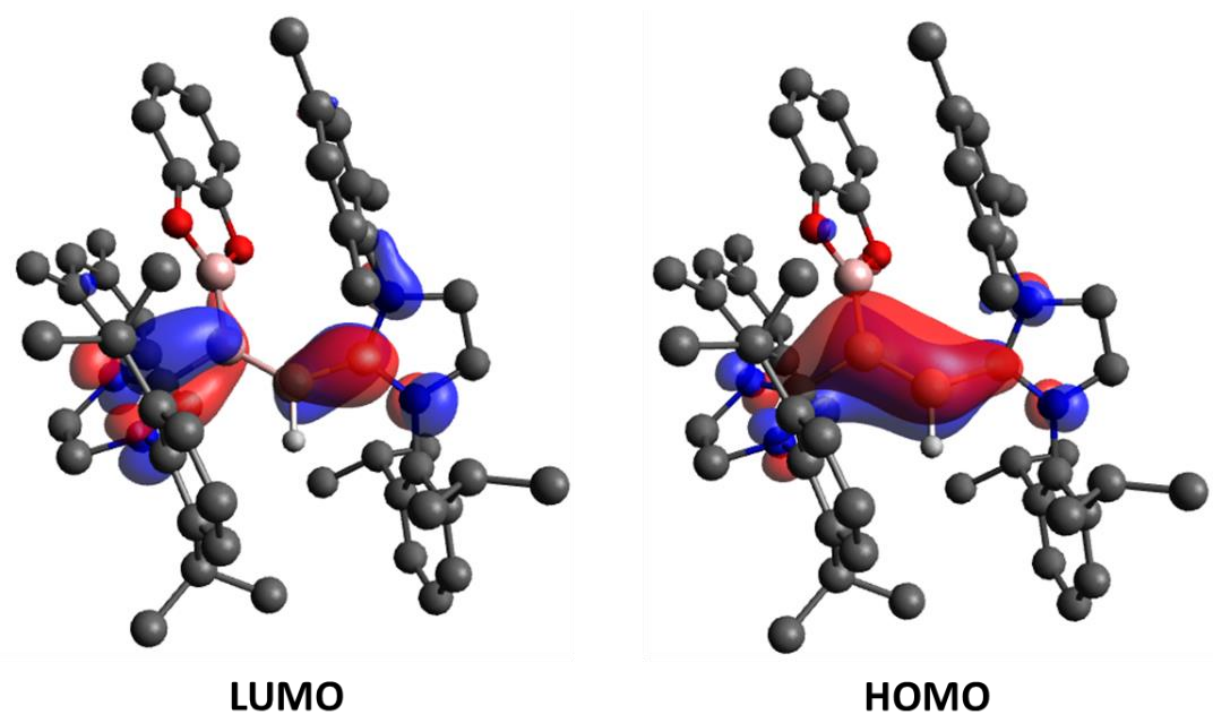

Figure S15. Frontier orbitals of compound 2.

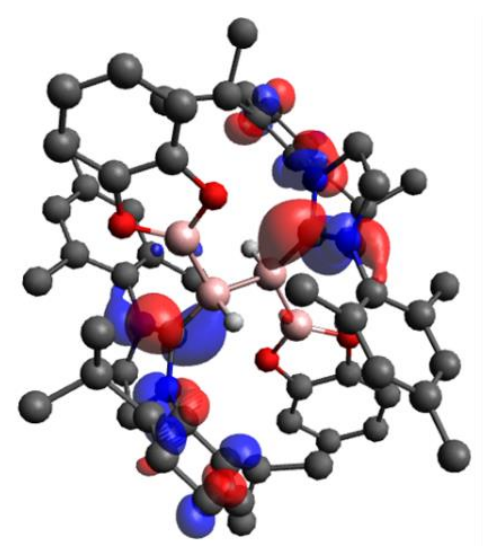

LUMO

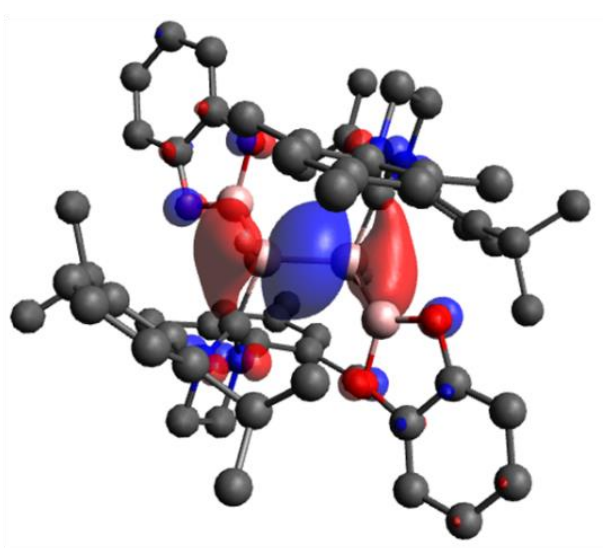

HOMO

Figure S16. Frontier orbitals of compound 3.

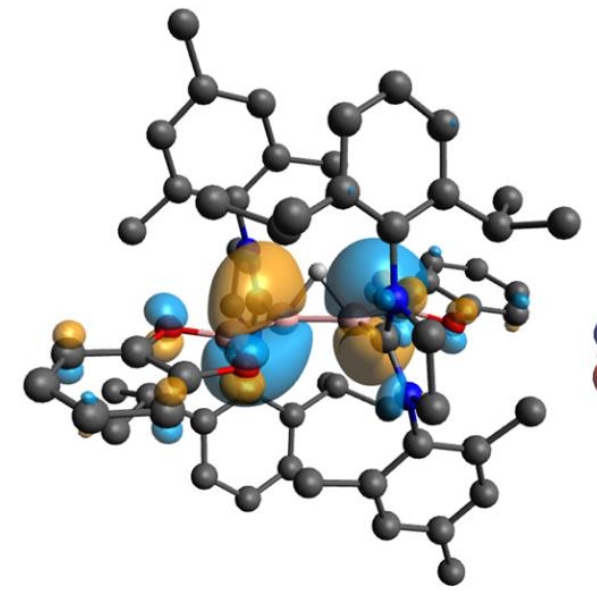

LUMO

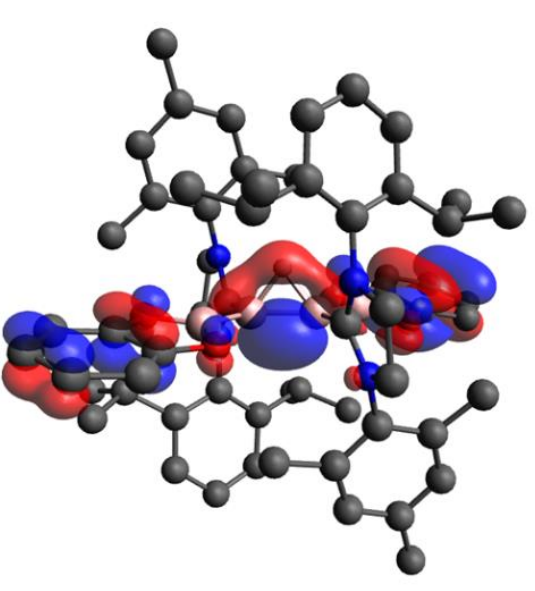

HOMO

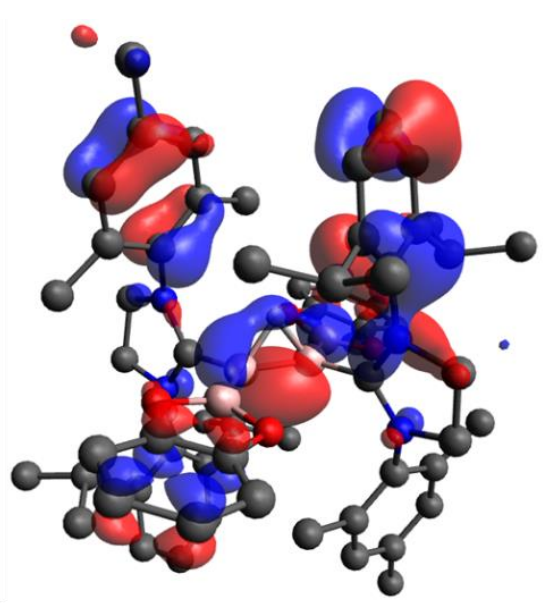

HOMO-8

Figure S17. LUMO, HOMO and HOMO-8 of compound 4. HOMO and HOMO-8 both show $3 \mathrm{c}-2 \mathrm{e}$ bonding across the central $\mathrm{B}-\mathrm{H}-\mathrm{B}$ unit. 


\section{Optimised Atomic Coordinates for Compound 2}

\begin{tabular}{|c|c|c|c|}
\hline & 1.0004809459 & -0.2478862485 & 23461 \\
\hline $\mathrm{H}$ & 2.0090377763 & 0.4018716011 & \\
\hline & -2.5460058226 & -1.0375868994 & .05244364 \\
\hline & -3.8288013221 & -1.3133915203 & 0.6702876027 \\
\hline & 466516 & 0.4663702762 & 0.0044927379 \\
\hline & 08803954 & -0.0854443996 & \\
\hline & 66113163 & -2.0260977510 & 1.34037248 \\
\hline & -4.6156410833 & -2.4579439405 & 2.3164351339 \\
\hline & -1.9641141769 & -0.2384055758 & $0.0356857 \varepsilon$ \\
\hline & -6.0510390340 & -2.1554040805 & 0.7066353616 \\
\hline & -6.8439486907 & -2.7071630636 & 1.2013290705 \\
\hline & -6.2872268562 & -1.5866828882 & -0.5462660390 \\
\hline & 126 & -1.7 & \\
\hline & -5.2895020451 & -0.8623831839 & -1.214158755 \\
\hline & -5.4638612520 & -0.4129303347 & -2.1855506975 \\
\hline & 614 & -0.7 & 3556065 \\
\hline & 1.3543924032 & -1.75965 & 0.2897069 \\
\hline & 0.6599965453 & 2430925 & -0.1047354574 \\
\hline & 2.5242216818 & 1005270 & 1.0580783978 \\
\hline & 2.2862460716 & -3.9 & 191807 \\
\hline & 3.5039589130 & -4.0902907047 & 0.825008979 \\
\hline & 2.5168777606 & 0634481 & 0.8759040690 \\
\hline & & & 8043 \\
\hline & 1.8364647277 & -4.4652210702 & -0.8612764097 \\
\hline & 0.8087668486 & -4.9129647589 & 0.5058343978 \\
\hline & 200279 & -2.9 & -1.0 \\
\hline & 82897 & -3.6 & 212333 \\
\hline C & -2.6015060975 & -3.81925 & -1.5999010681 \\
\hline $\mathrm{H}$ & 9637 & -4.2 & -1.2825548245 \\
\hline & & & \\
\hline C & 3858226 & 82180 & -3.3143747221 \\
\hline $\mathrm{H}$ & 0994006 & -2.6 & -4.3554959485 \\
\hline C & -0.1 & & -2.4 \\
\hline & 1.1 & -2.0 & 105748 \\
\hline $\mathrm{H}$ & 1.30 & -1.0 & 231089 \\
\hline $\mathrm{H}$ & 1.11 & -1.9 & 170555 \\
\hline $\mathrm{H}$ & & & 394 \\
\hline & & $-4 .($ & 999 \\
\hline $\mathrm{H}$ & 8925747 & -5.0 & 0.9255327841 \\
\hline $\mathrm{H}$ & 2888091 & -4.1 & 0.9954166618 \\
\hline t & & & \\
\hline C & & -3 & 0257761 \\
\hline $\mathrm{H}$ & -4.3 & -2.8 & -3.7989202145 \\
\hline $\mathrm{H}$ & -4.0 & -4.6 & -3.8 \\
\hline & & & \\
\hline C & 3.6360400519 & -1.479893 & 1.3963593045 \\
\hline C & 3.6357596303 & -1.0 & 2.7312946397 \\
\hline C & & & \\
\hline $\mathrm{H}$ & & & 3972335 \\
\hline C & 4679 & -0.3 & 2.4520416196 \\
\hline $\mathrm{H}$ & 2423816 & 98220 & 2.8648271635 \\
\hline C & 5.9 & & 118 \\
\hline $\mathrm{H}$ & 6.83 & & \\
\hline C & 503272 & $-1.3^{3}-x-x$ & 5165092 \\
\hline C & 4.8397 & -1.8999589441 & -0.8407671839 \\
\hline $\mathrm{H}$ & & & -1.0778435475 \\
\hline & & & -1.8553013725 \\
\hline $\mathrm{H}$ & & & -1.7019387848 \\
\hline & & & \\
\hline
\end{tabular}




\begin{tabular}{|c|c|c|c|}
\hline 11 & 5.0915691471 & -1.1734963655 & -2.8756928596 \\
\hline C & 5.8630406285 & -3.0410337174 & -0.9951171422 \\
\hline $\mathrm{H}$ & 6.8850808413 & -2.6912217325 & \\
\hline H & 5.8250333752 & -3.4564483778 & -2.0067824601 \\
\hline $\mathrm{H}$ & 5.6758112763 & -3.8564022792 & -0.2906518543 \\
\hline C & 2.4276206788 & -1.1836211025 & \\
\hline $\mathrm{H}$ & 1.5732242872 & -1.4637346123 & 3.0262994 \\
\hline C & 2.0612487598 & 0.1323119306 & 4.3507413177 \\
\hline$\Pi$ & 2.8338062187 & 0.4461378635 & 5.05880065 \\
\hline $\mathrm{H}$ & 1.1332601384 & 0.0124693700 & 4.91593852 \\
\hline $\mathrm{H}$ & 1.9152774654 & 0.9371524467 & 3.62893124 \\
\hline & 2.6553068193 & -2.2983660394 & 4.6862923050 \\
\hline $\mathrm{H}$ & 3.4781949310 & -2.0467160297 & 5.36239713 \\
\hline $\mathrm{H}$ & 2.9016740106 & -3.2539934542 & 4.2165076 \\
\hline$\Pi$ & 1.7582626870 & 7700918 & \\
\hline $\mathrm{N}$ & -1.4884798223 & 2.7838081749 & 0.6152118019 \\
\hline C & 3082 & 233903 & 0.024483 \\
\hline v & 535 & 95980 & -0.5 \\
\hline C & -1.1774495468 & 4.2179351372 & 0.6403323732 \\
\hline $\mathrm{H}$ & -0.8 & 64978 & 60381 \\
\hline $\mathrm{H}$ & -2.05 & 70026 & $0.40 \varepsilon$ \\
\hline C & -2.5762375842 & 483020 & 1.4 \\
\hline C & 642797 & 18438 & 8520 \\
\hline 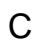 & 5258 & 784 & 17533 \\
\hline $\mathrm{H}$ & -3.279 & 1.2645474321 & 4.5746331 \\
\hline $\mathrm{C}$ & -4.760 & 7311 & 3.08629 \\
\hline C & -4.9 & & 1.7 \\
\hline $\mathrm{H}$ & 0476 & 289597 & 1.3 \\
\hline C & 9270 & 862629 & \\
\hline C & 886581 & 2.9511778876 & -0 \\
\hline $\mathrm{H}$ & 0569 & 681 & 505566 \\
\hline $\mathrm{H}$ & 34337 & 44899 & -0.7337359 \\
\hline $\mathrm{H}$ & -3.53 & 78089 & -1.1 \\
\hline C & 46388 & 1.7859929380 & 33 \\
\hline $\mathrm{H}$ & -0.9 & & 4.3 \\
\hline $\mathrm{H}$ & -0.3 & 855 & 3.1 \\
\hline $\mathrm{H}$ & 93173 & 31497 & 3605849 \\
\hline C & & & \\
\hline $\mathrm{H}$ & -5.6 & 2628 & 5.0 \\
\hline $\mathrm{H}$ & 63759 & 5888 & 3.6 \\
\hline $\mathrm{H}$ & -6.7 & & \\
\hline C & & & \\
\hline C & 7629 & 54494 & -1.2793487 \\
\hline C & 3.64 & & -2.3 \\
\hline $\mathrm{H}$ & & & \\
\hline C & 3.2 & & -3.5 \\
\hline $\mathrm{H}$ & 3.98 & & -4.36 \\
\hline C & $1.8 \mathrm{~s}$ & 96 & -3.8 \\
\hline $\mathrm{H}$ & & & \\
\hline C & 0.92 & & 28 \\
\hline C & & & -3.2 \\
\hline $\mathrm{H}$ & -1.1515209061 & 650027 & -2.3627208884 \\
\hline C & & & \\
\hline 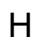 & & & 47802305 \\
\hline $\mathrm{H}$ & -1.9966259461 & 0.9072865106 & -4.1133682180 \\
\hline $\mathrm{H}$ & -0.7509 & 503336 & -3.153722945 \\
\hline C & & & -4.22561407 \\
\hline 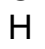 & -0.660 & 4.4065157329 & -38123748 \\
\hline $\mathrm{H}$ & -1.9893326141 & 3.4179685215 & -4.43491675 \\
\hline$\Pi$ & & 3.3311417231 & \\
\hline & 3.1839761445 & 3.4287952491 & 0.0859029 \\
\hline
\end{tabular}




$\begin{array}{ll}\mathrm{H} & 2.3289340561 \\ \mathrm{C} & 3.6504685923 \\ \mathrm{H} & 3.8992984421 \\ \mathrm{H} & 2.8884609320 \\ \mathrm{H} & 4.5455094925 \\ \mathrm{C} & 4.2906161080 \\ \mathrm{H} & 5.2064749825 \\ \mathrm{H} & 3.9729269206 \\ \mathrm{H} & 4.5476260267 \\ \mathrm{C} & -0.0828646465 \\ \mathrm{H} & -0.4711140562 \\ \mathrm{H} & 0.7266107693\end{array}$

3.3735255684

4.8970979677

5.2720658836

5.5551659108

4.9966134513

2.5521603599

2.5818978806

1.5136490143

2.9052002676

4.3251384491

4.6671240301

4.9946537599
0.7634862291

0.0140772215

1.0118298126

$-0.4124379046$

$-0.6073660513$

0.6896310299

0.0919234247

0.7707591877

1.6929641246

$-0.4107395686$

$-1.3773883756$

$-0.1185374944$

\section{Optimised Atomic Coordinates for Compound 3}

O $\quad-1.9395098920$

H $\quad 0.7358014768$

C $\quad-3.3728630015$

N 1.2943625989

B -1.5505590823

O -2.4960881502

C $\quad-4.4479792649$

$\mathrm{H} \quad-4.7141118070$

N $\quad-0.4576256583$

B -0.1164074360

C $\quad-3.0267856264$

C $\quad-3.7387547556$

H $\quad-3.4611952772$

C $\quad-4.8307405111$

$\mathrm{H} \quad-5.4129562038$

C $\quad-5.1789571379$

H $\quad-6.0290091752$

C $\quad 0.1956809690$

C $\quad 1.3798523200$

H 2.3819080020

H $\quad 1.1574304661$

C $\quad 0.3255292600$

H $\quad-0.3100506457$

H $\quad 0.7672023602$

C $\quad-1.7964849769$

C $\quad-3.3553945602$

H $\quad-3.5405014356$

C $\quad-2.0386238518$

C $\quad-4.4153622105$

C $\quad-4.1285354052$

H $\quad-4.9312875304$

C $\quad-2.8398505708$

C $\quad-0.9463540552$

H $\quad-0.8533706927$

H $\quad-1.1846444677$

H $\quad 0.0176847160$

C $\quad-5.8300128269$

H $\quad-6.3549805363$

$\mathrm{H} \quad-6.4074703115$

H $\quad-5.8566069979$

C $\quad-2.5902138633$

H $\quad-3.5017486350$

H $\quad-1.8198656876$

H $\quad-2.2773960643$

C $\quad 2.1330045384$
0.9899893833

1.3720561374

2.5800398547

2.3680996837

1.4023388588

2.3843283633

3.4449647190

4.0956516826

1.4417318367

0.9314043492

1.7345498753

1.7180257508

1.0604921549

2.5922153968

2.6116739571

3.4368542158

4.1025954507

1.5886167345

2.8226067758

2.6641990231

3.8934134571

1.9629656242

2.5265888608

1.1286265357

0.9733746901

$-0.7080011521$

$-1.7330039476$

$-0.3366608296$

0.1869906224

1.5100514825

2.2428880958

1.9274891396

$-1.3419030697$

$-1.5242445391$

$-2.2987873227$

$-1.0212357258$

$-0.2430275095$

0.4966402455

$-0.3652954693$

$-1.1969589399$

3.3899276911

3.9673646437

3.8044230542

3.5530009346

3.0571922070
2.0848030142

0.7771405198

1.4018131053

$-1.6195428666$

0.7724888563

0.3701325522

1.4863591340

0.6610070304

$-2.5777191057$

0.0339652372

2.4531312060

3.6383144873

4.4547372177

3.7376462722

4.6529703337

2.6828537421

2.7895073500

$-1.3900931425$

$-3.0202608926$

$-3.4183870266$

$-3.0787458646$

$-3.7113314697$

$-4.3956395220$

$-4.2655144919$

$-2.8508735384$

$-3.5895464365$

$-3.9000384382$

$-3.2885082385$

$-3.5228574715$

$-3.1773323511$

$-3.1689073102$

$-2.8557887162$

$-3.5391349254$

$-4.6165741339$

$-3.0699780438$

$-3.1614886426$

$-3.8227239918$

$-4.4338378824$

$-2.9000059488$

$-4.3539764320$

$-2.5835516175$

$-2.7514070249$

$-3.2409352128$

$-1.5547342058$

$-0.6624477841$ 


\begin{tabular}{|c|c|c|c|}
\hline C & 1.6315766685 & 4.1815996764 & 0.0353395848 \\
\hline & 2.5052888802 & 4.8953090789 & 0.8597268201 \\
\hline $\mathrm{H}$ & 2.1344793182 & 5.7571865069 & 1.4037838671 \\
\hline $\mathrm{C}$ & 3.8402076416 & 4.5376161794 & 0.98609016 \\
\hline $\mathrm{H}$ & 4.5016414693 & 5.1112748332 & 1.6283304468 \\
\hline & 4.3236355434 & 3.4504093916 & 0.2759675 s \\
\hline $\mathrm{H}$ & 5.3698251920 & 3.1790742067 & 0.366611079 \\
\hline C & 3.4927503364 & 2.6957947813 & -0.55848719 \\
\hline & 4.1048052523 & 1.5353016281 & -1.3298830105 \\
\hline $\mathrm{H}$ & 3.2959335155 & 1.0082696671 & -1.83495166 \\
\hline C & 4.7932808450 & 0.5275510695 & -0.39326774 \\
\hline $\mathrm{H}$ & 5.1623353802 & -0.3287053684 & -0.9620308929 \\
\hline $\mathrm{H}$ & 4.1098423050 & 1499554 & 0.373509386 \\
\hline $\mathrm{H}$ & 5.6522012414 & 047878 & 0.1164 \\
\hline 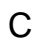 & 902347 & 76635 & -2.39 \\
\hline $\mathrm{H}$ & 5.4707167956 & 1.1888855161 & -2.9910974921 \\
\hline $\mathrm{H}$ & 5.9628928223 & 67034 & 801800 \\
\hline-1 & 776916 & 9303 & \\
\hline C & 0.2013939735 & 38550 & -0.1025428730 \\
\hline $\mathrm{H}$ & -0.3807290713 & 77213 & -0.6219226685 \\
\hline C & 387256 & 4560 & 1.2 \\
\hline $\mathrm{H}$ & 392542 & 48076 & 1.12 \\
\hline $\mathrm{H}$ & 5075122 & 00647 & 30822 \\
\hline $\mathrm{H}$ & 273420 & 1696 & 1.8 \\
\hline C & 0.1498603892 & 75897 & -0.9 \\
\hline $\mathrm{H}$ & 3574071 & 4827 & -1.0 \\
\hline $\mathrm{H}$ & 0861181 & 37619 & -1.9305403807 \\
\hline H & 0.6866954564 & 920 & $-0.4 t$ \\
\hline $\mathrm{O}$ & 1.9397759782 & 380 & -2.08 \\
\hline $\mathrm{H}$ & 104530 & -1 & -0.7 \\
\hline C & 114145 & 81845 & -1.4 \\
\hline $\mathrm{N}$ & 5713 & -2 & \\
\hline B & 4009 & 7395 & -0.7 \\
\hline 0 & 2.4961123812 & -2.3842758795 & -0.3697936328 \\
\hline C & 4.4 & & \\
\hline $\mathrm{H}$ & 39343 & 763 & -0.6 \\
\hline $\mathrm{N}$ & 334176 & 501502 & 502876 \\
\hline B & 0.1164322179 & 27505 & -0.03 \\
\hline C & & & \\
\hline C & 5375 & 49600 & -3.6 \\
\hline $\mathrm{H}$ & 812669 & -1.0 & -4.4 \\
\hline C & & & \\
\hline $\mathrm{H}$ & 345944 & 701 & -4.6 \\
\hline C & 5.1792521966 & 49380 & -2.68 \\
\hline $\mathrm{H}$ & 09635 & -4.1 & -2.7 \\
\hline C & & & \\
\hline C & -1.3 & $-2 . \varepsilon$ & \\
\hline $\mathrm{H}$ & 3315835 & 97694 & \\
\hline $\mathrm{H}$ & & & 3.0782351133 \\
\hline C & & & \\
\hline $\mathrm{H}$ & & -2 & \\
\hline $\mathrm{H}$ & -0.7669553614 & 64139 & 16556 \\
\hline C & & & \\
\hline C & & & \\
\hline $\mathrm{H}$ & 3.54 & 158741 & 3.9 \\
\hline C & 2.0386360229 & 0.3365067814 & 4832402 \\
\hline C & & & \\
\hline 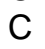 & 4.1284709620 & -1.5103173623 & \\
\hline $\mathrm{H}$ & 4.9311941570 & -2.2431853187 & 3.1692218531 \\
\hline C & & -1.9276625986 & 2.050009 \\
\hline & 0.9464918317 & 1.3420047352 & 3.53864075 \\
\hline
\end{tabular}




$\begin{array}{lrrr}\mathrm{H} & 0.8545244949 & 1.5259973275 & 4.6158702647 \\ \mathrm{H} & 1.1842131301 & 2.2981773877 & 3.0677435507 \\ \mathrm{H} & -0.0178550074 & 1.0206966001 & 3.1623109628 \\ \mathrm{C} & 5.8299194800 & 0.2426078566 & 3.8234980828 \\ \mathrm{H} & 6.3546259346 & -0.4970059582 & 4.4349034495 \\ \mathrm{H} & 6.4076534597 & 0.3646302248 & 2.9009207639 \\ \mathrm{H} & 5.8564752857 & 1.1966365387 & 4.3545763549 \\ \mathrm{C} & 2.5901181458 & -3.3900871322 & 2.5835807646 \\ \mathrm{H} & 3.5021834123 & -3.9672882305 & 2.7493345235 \\ \mathrm{H} & 1.8213274338 & -3.8050180081 & 3.2425462240 \\ \mathrm{H} & 2.2751880142 & -3.5530339659 & 1.5554078907 \\ \mathrm{C} & -2.1331160308 & -3.0569784088 & 0.6621267429 \\ \mathrm{C} & -1.6318092842 & -4.1812941030 & -0.0358895792 \\ \mathrm{C} & -2.5056276806 & -4.8948395294 & -0.8603039461 \\ \mathrm{H} & -2.1349078936 & -5.7566548955 & -1.4045197303 \\ \mathrm{C} & -3.8405410973 & -4.5370611308 & -0.9864891301 \\ \mathrm{H} & -4.5020647105 & -5.1105875537 & -1.6287555454 \\ \mathrm{C} & -4.3238510403 & -3.4499473511 & -0.2761442299 \\ \mathrm{H} & -5.3700360162 & -3.1785514484 & -0.3666433377 \\ \mathrm{C} & -3.4928535207 & -2.6955062475 & 0.5583582515 \\ \mathrm{C} & -4.1048010295 & -1.5351633011 & 1.3300641849 \\ \mathrm{H} & -3.2958561498 & -1.0082177813 & 1.8351063510 \\ \mathrm{C} & -4.7934481391 & -0.5272285937 & 0.3937737070 \\ \mathrm{H} & -5.1623221719 & 0.3289527116 & 0.9627641844 \\ \mathrm{H} & -4.1101787770 & -0.1587380619 & -0.3731130312 \\ \mathrm{H} & -5.6525213407 & -0.9738541882 & -0.1157993056 \\ \mathrm{C} & -5.0983092455 & -2.0302567823 & 2.3997410834 \\ \mathrm{H} & -5.4704805709 & -1.1890948216 & 2.9915518701 \\ \mathrm{H} & -5.9627578821 & -2.5228734177 & 1.9442581145 \\ \mathrm{H} & -4.6421725416 & -2.7499293346 & 3.0857111225 \\ \mathrm{C} & -0.2016390731 & -4.6884344884 & 0.1017890590 \\ \mathrm{H} & 0.3805343897 & -3.9299757339 & 0.6212542330 \\ \mathrm{C} & 0.4773920077 & -4.9142897763 & -1.2594670981 \\ \mathrm{H} & 1.5345013692 & -5.1537566345 & -1.1217128037 \\ \mathrm{H} & 0.0251158400 & -5.7443121546 & -1.8097709338 \\ \mathrm{H} & 0.4168269380 & -4.0239250987 & -1.8872893728 \\ \mathrm{C} & -0.1500723972 & -5.9796316499 & 0.9425696338 \\ \mathrm{H} & 0.8851455986 & -6.3031785327 & 1.0879023225 \\ \mathrm{H} & -0.6001744854 & -5.8441619670 & 1.9296509132 \\ \mathrm{H} & -0.6870074381 & -6.7955165405 & 0.4495589211\end{array}$

\section{Optimised Atomic Coordinates for Compound 4}

$\begin{array}{cl}\text { B } & 1.6919739864 \\ \text { O } & 2.6776778175 \\ \text { C } & 3.4355328709 \\ \text { B } & 0.4888649262 \\ \text { H } & 0.7311027747 \\ \text { O } & 1.8529670449 \\ \text { C } & 5.1077525609 \\ \text { H } & 5.9710792466 \\ \text { C } & 4.5892122484 \\ \text { H } & 5.0558399752 \\ \text { C } & 3.4786301740 \\ \text { H } & 3.0726847112 \\ \text { C } & 2.9254483074 \\ \text { C } & 4.5345041518 \\ \text { H } & 4.9335442859 \\ \mathrm{C} & 0.2884111217 \\ \text { N } & 0.9133184640\end{array}$

1.2689272255

2.1927187097

2.4495737117

0.6278469079

$-0.7109524898$

0.9696948394

3.3357683459

3.9711972626

2.5977672753

2.6734291331

1.7617882614

1.1867512545

1.7090414966

3.2753402088

3.8436043666

1.2867553961

0.8575997341
0.7841817464

0.4155285255

1.5379933991

$-0.2265627761$

$-0.4551056233$

2.1453688538

2.9587145246

3.1208909133

4.0264191321

5.0021263718

3.8630392886

4.6871786186

2.5968525555

1.6835106066

0.8518049156

$-1.6819723308$

$-2.7986875850$ 


\begin{tabular}{|c|c|c|c|}
\hline & -0.4950542553 & 2.6236411779 & -3.4352322823 \\
\hline $\mathrm{H}$ & -0.0285807914 & 3.6013357797 & 360565 \\
\hline & .5007149021 & 3319667 & \\
\hline & -0.5548577813 & 36697346 & 9851244 \\
\hline C & 3398347991 & 808556064 & 11623829 \\
\hline & -0.2646549646 & 7416336816 & 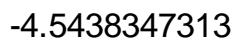 \\
\hline & 1.1321411455 & 1.8172630723 & -4.6790659563 \\
\hline C & 2.1467457200 & 1031743418 & -2.9183774892 \\
\hline & 2.1333300935 & 2711373341 & 3772012 \\
\hline 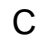 & 3.3592582100 & -1.9257601219 & 3259 \\
\hline $\mathrm{H}$ & 3.3462580987 & -2.9941575758 & -3.5597205009 \\
\hline & 4.5747016591 & -1.2558178179 & -3.3167674868 \\
\hline C & 4.5497553406 & 0.12650 & 9011340 \\
\hline $\mathrm{H}$ & 5.4855380967 & 0.6784 & 5714749 \\
\hline 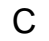 & 3.3623291100 & 0.82695 & 7359465 \\
\hline C & 3.4097693017 & 2.32722 & Q762 \\
\hline $\mathrm{H}$ & 3.06227 & 2.6 & 693584 \\
\hline & 2.8025 & 2.8 & 1870 \\
\hline & 4.4325965068 & 2.6872242008 & 8528272 \\
\hline C & 0.8704 & -2.0 & 1683 \\
\hline $\mathrm{H}_{\text {- }}$ & -0.02 & -1.4 & \\
\hline$\Pi$ & 0.83910 & -2.886 & -2 \\
\hline$\Pi$ & 0.8385 & & -4 \\
\hline C & 5.8839 & -1.9 & 8756 \\
\hline $\mathrm{H}$ & 5.73301 & -3.0382 & 3493532 \\
\hline $\mathrm{H}$ & $6.5197 \varepsilon$ & $-1.8 s$ & 777136 \\
\hline $\mathrm{H}$ & & & \\
\hline C & -1.325 & & 7100 \\
\hline C & -0.69 & $4.2 \varsigma$ & 107 \\
\hline C & -1. & 5.15 & \\
\hline 1 & -1.02 & & 53418 \\
\hline 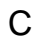 & -2.84 & 4.9 & 5273567 \\
\hline 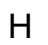 & -3.42 & & 899657 \\
\hline C & & & 17040 \\
\hline$\Pi$ & & & 9778 \\
\hline C & $-2.7207 t$ & 2.9 & 19332 \\
\hline C & -3.485 & & 08291 \\
\hline H & & & \\
\hline C & -4.43 & 1.1 & 661 \\
\hline 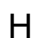 & -4.89 & 0.2 & 50772 \\
\hline $\mathrm{H}$ & & & \\
\hline $\mathrm{H}$ & & & \\
\hline C & -4.2805 & 2.5 & 37058 \\
\hline $\mathrm{H}$ & -3.6 & & 99733 \\
\hline 1 & & & \\
\hline $\mathrm{H}$ & -4.76 & & \\
\hline C & 0.75 & & -0 . \\
\hline $\mathrm{H}$ & & & \\
\hline C & & & 972 \\
\hline$H$ & & & \\
\hline $\mathrm{H}$ & & & \\
\hline $\mathrm{H}$ & 77 & 87 & $20<94$ \\
\hline C & & & \\
\hline to & & & -2 \\
\hline $\mathrm{H}$ & 54 & 15 & -2 \\
\hline $\mathrm{H}$ & 9 & 6. & 8134 \\
\hline B & & & \\
\hline 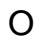 & & & -0.2107615156 \\
\hline C & -3.6210 & -2.3047349729 & -1.274739591 \\
\hline D & & & \\
\hline & ה" & -1.0711321888 & -2.0951817 \\
\hline
\end{tabular}




\begin{tabular}{|c|c|c|c|}
\hline & -5.4222537944 & -3.1784709446 & 89874670 \\
\hline H & -6.3462587963 & -3.7420724586 & \\
\hline & 92623410 & -2.6181693692 & 30062373 \\
\hline & -5.3888441384 & -2.7551762906 & 451336214 \\
\hline & -3.6881955406 & -1.8828030878 & -3.6626701911 \\
\hline & -3.2615182218 & -1.4464346663 & 5836056 \\
\hline & -3.0836699432 & -1.7440976504 & -2.427489077 \\
\hline & -4.7970305524 & -3.0306652785 & -1.295065134 \\
\hline & -5.2139304914 & -3.4617785152 & 5000 \\
\hline & -0.2362647640 & -1.3003853764 & 1.64771924 \\
\hline & -0.9928266939 & -1.0195380883 & 2.724853621 \\
\hline & 0.6639013598 & -2.5421484170 & 3.4192615184 \\
\hline & 0.4399847375 & -3.6051979002 & 3.5223947060 \\
\hline & 1.6239283910 & -2.3499314585 & 3.896 \\
\hline & 0.7288088550 & -2.1868229823 & 1.9810598440 \\
\hline & -0.4529096294 & -1.6401635001 & 3.952 \\
\hline & -0.0822824198 & -0.8549228595 & 4.61570243 \\
\hline & -1.2328569353 & -2.1886350862 & 4.47 \\
\hline & -2.2542394020 & -0.3103787631 & 2.8067811757 \\
\hline & -2.296 & 9305 & 48578 \\
\hline & -3.545 & 1.6950476857 & 3.12330230 \\
\hline & -3.5758990039 & 2.7746060955 & 3.2377563659 \\
\hline & -4.7321355775 & 092383 & 516829 \\
\hline & -4.6 & -0.4 & 3.0 \\
\hline $\mathrm{H}$ & -5.562 & -1.0078409119 & 3.08875868 \\
\hline C & -3.43 & -1.0798736618 & 2.87 \\
\hline & 5658 & 6127636 & 73415 \\
\hline$H$ & -2.6582773185 & -2.9999882778 & 2.2199394 \\
\hline $\mathrm{H}$ & -3.3071144373 & -2.9995772606 & 3.86377706 \\
\hline $\mathrm{H}$ & & 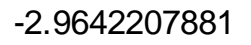 & 2.4870426120 \\
\hline C & 8982 & 3097 & 348 \\
\hline $\mathrm{H}$ & 84938 & 429682 & 3.215603436 \\
\hline $\mathrm{H}$ & 4604 & 20745 & 2.0 \\
\hline $\mathrm{H}$ & & & \\
\hline C & -6.0 & 60969 & 82691 \\
\hline $\mathrm{H}$ & 60558 & 06261 & 69386 \\
\hline $\mathrm{H}$ & -6.68 & 1.5 & 748 \\
\hline $\mathrm{H}$ & & & \\
\hline C & 3989986 & -3.0 & 1.113556357 \\
\hline C & 0.9188026184 & -4.13 & 8248053 \\
\hline C & & & 774 \\
\hline $\mathrm{H}$ & 1.2 & -5 & -0.8 \\
\hline C & 75831 & 644143 & -0.393985783 \\
\hline $\mathrm{H}$ & 3.71 & -5.4 & -0.9 \\
\hline & & & \\
\hline $\mathrm{H}$ & 4.765981 & 560654 & 0.2 \\
\hline C & 2.9295498541 & 180596 & 1.0739982390 \\
\hline 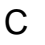 & & & 3253819 \\
\hline $\mathrm{H}$ & 2.9 & -1.1 & \\
\hline C & $4.5 \varepsilon$ & & \\
\hline $\mathrm{H}$ & 5.02 & -0 & \\
\hline $\mathrm{H}$ & 5.4 & -1. & 427 \\
\hline $\mathrm{H}$ & & & \\
\hline$C$ & 4.46 & -2 & 30 \\
\hline $\mathrm{H}$ & 3.8368516579 & -3.0352807039 & 3.6835209479 \\
\hline $\mathrm{H}$ & 5.26 & 83706 & 2.656038 \\
\hline to & 4.9272047747 & & 3.644840036 \\
\hline C & -0.5516750979 & -4.5099422752 & 0.60853098 \\
\hline $\mathrm{H}$ & 5176136 & 4826693 & 1.01116077 \\
\hline 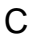 & & -4.8724059983 & \\
\hline & -0.84 & -5.8116778949 & -1.1349443 \\
\hline
\end{tabular}




$\begin{array}{llll}\text { H } & -2.2997837792 & -4.9987561586 & -0.5837285098 \\ \text { H } & -1.0781530685 & -4.0994129948 & -1.4829465390 \\ \mathrm{C} & -0.7123680124 & -5.6782983562 & 1.6037927854 \\ \mathrm{H} & -0.2767062309 & -5.4564787008 & 2.5810274423 \\ \mathrm{H} & -1.7702163874 & -5.9116245997 & 1.7541574573 \\ \mathrm{H} & -0.2233933777 & -6.5817558305 & 1.2299270240\end{array}$

\section{Crystallographic Details}

The crystal data of 2, 3 and $\mathbf{4}$ were collected on a Bruker D8 Quest diffractometer with a CMOS area detector and multi-layer mirror monochromated $\mathrm{Mo}_{\mathrm{K \alpha}}$ radiation. The structures were solved using intrinsic phasing method, ${ }^{7}$ refined with the SheIXL program ${ }^{8}$ and expanded using Fourier techniques. All non-hydrogen atoms were refined anisotropically. Hydrogen atoms were included in structure factor calculations. All hydrogen atoms were assigned to idealized geometric positions.

Crystal data for 2: $\mathrm{C}_{54} \mathrm{H}_{69} \mathrm{~B}_{3} \mathrm{~N}_{4} \mathrm{O}_{2}, \quad M_{\mathrm{r}}=838.56$, red block, $0.223 \times 0.201 \times 0.189 \mathrm{~mm}^{3}$, monoclinic space group $P 2_{1} / c, \quad a=19.5024(6) \AA, \quad b=11.4232(3) \AA, \quad c=23.2030(7) \AA$, $\beta=110.9810(10)^{\circ}, \quad V=4826.4(2) \AA^{3}, \quad Z=4, \quad \rho_{\text {calcd }}=1.154 \mathrm{~g} \cdot \mathrm{cm}^{-3}, \quad \mu=0.069 \mathrm{~mm}^{-1}$, $F(000)=1808, \quad T=100(2) \mathrm{K}, \quad R_{1}=0.0536, \quad w R^{2}=0.1063,9486$ independent reflections $\left[2 \theta \leq 52.042^{\circ}\right]$ and 586 parameters.

All hydrogen atoms except $\mathrm{H}_{1} \_1$ were assigned to idealized positions. The coordinates of H1_1 were refined freely. CCDC number: 1942654.

Crystal data for 3: $\mathrm{C}_{66} \mathrm{H}_{80} \mathrm{~B}_{4} \mathrm{~N}_{4} \mathrm{O}_{4}, M_{\mathrm{r}}=1036.58$, colorless block, $0.255 \times 0.174 \times 0.132 \mathrm{~mm}^{3}$, triclinic space group $P \overline{1}, \quad a=10.3336(4) \AA, \quad b=12.7293(5) \AA, \quad c=13.7552(6) \AA$, $\alpha=100.879(2)^{\circ}, \quad \beta=108.559(2)^{\circ}, \quad Y=113.0500(10)^{\circ}, \quad V=1472.97(11) \AA^{3}, \quad Z=1$, $\rho_{\text {calcd }}=1.169 \mathrm{~g} \cdot \mathrm{cm}^{-3}, \quad \mu=0.071 \mathrm{~mm}^{-1}, \quad F(000)=556, \quad T=100(2) \mathrm{K}, \quad R_{1}=0.0523$, $w R^{2}=0.1003,6009$ independent reflections $\left[2 \theta \leq 52.744^{\circ}\right]$ and 363 parameters.

All hydrogen atoms except $\mathrm{H} 1$ were assigned to idealized positions. The coordinates of $\mathrm{H} 1$ were refined freely. CCDC number: 1942655.

Crystal data for 4: $\quad \mathrm{C}_{122} \mathrm{H}_{146} \mathrm{~B}_{8} \mathrm{~F}_{6} \mathrm{~N}_{8} \mathrm{O}_{14} \mathrm{~S}_{2}, \quad M_{\mathrm{r}}=2213.06$, colorless plate, $0.256 \times 0.245 \times 0.089 \mathrm{~mm}^{3}$, monoclinic space group $C 2 / c, a=20.3578(7) \AA, b=15.3176(5) \AA$, $c=19.7253(6) \AA, \quad \beta=98.6750(10)^{\circ}, \quad V=6080.6(3) \AA^{3}, \quad Z=2, \quad \rho_{\text {calcd }}=1.209 \mathrm{~g} \cdot \mathrm{cm}^{-3}$, 
$\mu=0.116 \mathrm{~mm}^{-1}, F(000)=2344, T=100(2) \mathrm{K}, R_{1}=0.1014, w R^{2}=0.2333,5994$ independent reflections $\left[2 \theta \leq 52.044^{\circ}\right]$ and 409 parameters.

All hydrogen atoms except H1_1 were assigned to idealized positions. The coordinates of $\mathrm{H} 1$ 1 were refined freely. The unit cell contains three pentane molecules which have been treated as a diffuse contribution to the overall scattering without specific atom positions by SQUEEZE/PLATON. ${ }^{9}$ The disordered triflate anion was restrained to an idealized geometry, and the displacement parameters of the atoms were restrained to the same value with similarity restraint SIMU and RIGU. The Uii displacement parameters of atoms of the disordered triflate anion were restrained with the ISOR keyword to approximate isotropic behavior. CCDC number: 1942656.

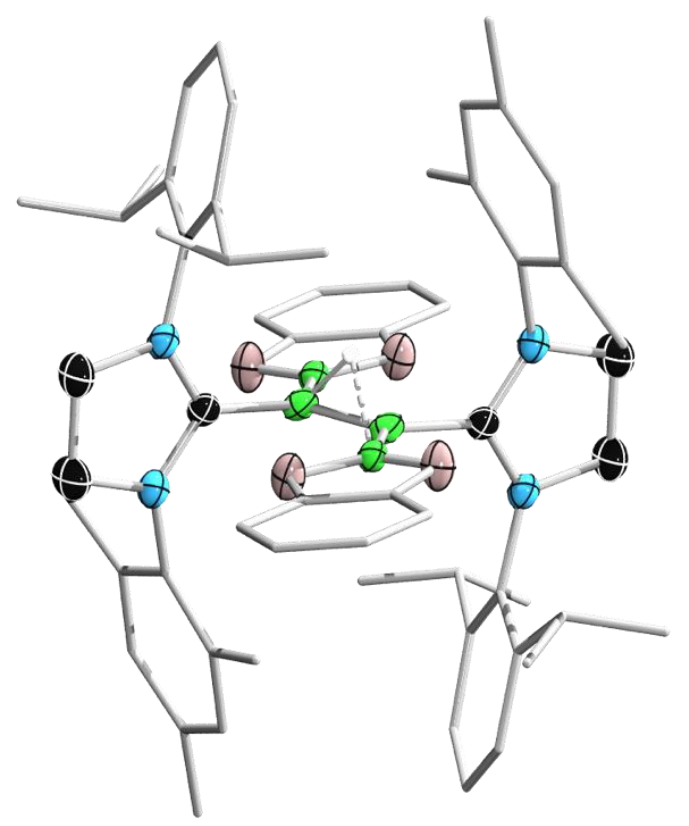

Figure S18. Crystallographically determined structure of compound 4, viewed adjacent to the catB-B-B-Bcat plane. 


\section{References}

1 Kuhn, K. M.; Grubbs, R. H.; A Facile Preparation of Imidazolinium Chlorides, Org. Lett. 2008, 10, 2075-2077.

2 Arrowsmith, M.; Böhnke, J.; Braunschweig, H.; Celik, M. A.; Dellermann, T.; Hammond, K.; Uncatalyzed Hydrogenation of First-Row Main Group Multiple Bonds, Chem. Eur. J. 2016, 22, 17169-17172.

3 Brückner, T.; Dewhurst, R. D.; Dellermann, T.; Müller, M.; Braunschweig, H.; Mild Synthesis of Diboryldiborenes by Diboration of B-B Triple Bonds, Chem. Sci. 2019, 10, 7375-7378.

4 Gaussian 09, Revision E.01, Frisch, M. J.; Trucks, G. W.; Schlegel, H. B.; Scuseria, G. E.; Robb, M. A.; Cheeseman, J. R.; Scalmani, G.; Barone, V.; Petersson, G. A.; Nakatsuji, H.; Li, X; Caricato, M.; Marenich, A; Bloino,J.; Janesko, B. G.; Gomperts, R.; Mennucci, B.; Hratchian, H. P.; Ortiz, J. V.; Izmaylov, A. F.; Sonnenberg, J. L.; Williams-Young, D.; Ding, F.; Lipparini, F.; Egidi, F.; Goings, J.; Peng, B.; Petrone, A.; Henderson, T.; Ranasinghe, D.; Zakrzewski, V. G.; Gao, J.; Rega, N.; Zheng, G.; Liang, W.; Hada, M.; Ehara, M.; Toyota, K.; Fukuda, R.; Hasegawa, J.; Ishida, M.; Nakajima, T.; Honda, Y.; Kitao, O.; Nakai, H.; Vreven, T.; Throssell, K.; Montgomery, J. A.; Peralta, J. E.; Ogliaro, F.; Bearpark, M.; Heyd, J. J.; Brothers, E.; Kudin, K. N.; Staroverov, V. N.; Keith, T.; Kobayashi, R.; Normand, J.; Raghavachari, K.; Rendell, A.; Burant, J. C.; lyengar, S. S.; Tomasi, J.; Cossi, M.; Millam, J. M.; Klene, M.; Adamo, C.; Cammi, R.; Ochterski, J. W.; Martin, R. L.; Morokuma, K.; Farkas, O.; Foresman, J. B.; Fox, D. J.; Gaussian, Inc., Wallingford CT, 2016.

5 NBO 6.0. Glendening, E. D.; Badenhoop, J. K.; Reed, A. E.; Carpenter, J. E.; Bohmann, J. A.; Morales, C. M.; Landis, C. R.; Weinhold, F. Theoretical Chemistry Institute, University of Wisconsin, Madison, WI, 2013; http://nbo6.chem.wisc.edu/

6 Hanwell, M. D.; Curtis, D. E.; Lonie, D. C.; Vandermeersch, T.; Zurek, E.; Hutchison, G. R.; Avogadro: An Advanced Semantic Chemical Editor, Visualization and Analysis Platform; J. Cheminformatics, 2012, 4:17.

7 Sheldrick, G.; Crystal Structure Refinement with SHELXL, Acta Cryst. 2015, A71, 3-8.

8 Sheldrick, G.; A Short History of SHELX, Acta Cryst. 2008, A64, 112-122.

9 Spek, A. L.; PLATON SQUEEZE: A Tool for the Calculation of the Disordered Solvent Contribution to the Calculated Structure Factors, Acta Cryst. 2015, C71, 9-18. 